\title{
Bulk Viscosity Effects in Event-by-Event Relativistic Hydrodynamics
}

\author{
Jacquelyn Noronha-Hostler, ${ }^{1}$ Gabriel S. Denicol, ${ }^{2}$ Jorge Noronha, ${ }^{1}$ Rone P. G. Andrade, ${ }^{1}$ and Frédérique Grassi ${ }^{1}$ \\ ${ }^{1}$ Instituto de Física, Universidade de São Paulo, C.P. 66318, 05315-970 São Paulo, SP, Brazil \\ ${ }^{2}$ Department of Physics, McGill University, 3600 University Street, Montreal, Quebec, H3A 2T8, Canada
}

(Dated: May 13, 2013)

Bulk viscosity effects on the collective flow harmonics in heavy ion collisions are investigated, on an event by event basis, using a newly developed $2+1$ Lagrangian hydrodynamic code named v-USPhydro which implements the Smoothed Particle Hydrodynamics (SPH) algorithm for viscous hydrodynamics. A new formula for the bulk viscous corrections present in the distribution function at freeze-out is derived starting from the Boltzmann equation for multi-hadron species. Bulk viscosity is shown to enhance the collective flow Fourier coefficients from $v_{2}\left(p_{T}\right)$ to $v_{5}\left(p_{T}\right)$ when $p_{T} \sim 1-3 \mathrm{GeV}$ even when the bulk viscosity to entropy density ratio, $\zeta / s$, is significantly smaller than $1 /(4 \pi)$.

PACS numbers: 25.75.-q,12.38.Mh, 24.10.Nz, 25.75.Ld 


\section{INTRODUCTION}

One of the most important discoveries that stemmed from the relativistic heavy ion collision program was the discovery that the Quark-Gluon Plasma (QGP) formed in these reactions behaves as a relativistic fluid for which viscous effects appear to be very small [1]. The large degree of collectivity evidenced by the Fourier harmonics of the flow are compatible [2] with viscous hydrodynamic calculations in which the shear viscosity to entropy density ratio, $\eta / s$, nears the uncertainty principle estimate $\sim 1 /(4 \pi)[3$, 4]. Since $\eta / s$ becomes much larger than $1 /(4 \pi)$ in the high temperature perturbative regime [5, [6] and also at sufficiently low temperatures [7], it was suggested that in QCD this quantity should have a minimum $\sim 1 /(4 \pi)$ in between these different temperature regimes [8, 9]. Further support to this idea appeared in [10] where it was shown that heavy resonances may already considerably lower $\eta / s$ in the hadronic phase.

While relativistic hydrodynamical studies that include shear viscous corrections are currently considered to be the state of the art in the field [11, 12] there is a priori no reason that effects from bulk viscosity $\zeta$ should not also be included in the description of the time evolution of the QGP. High temperature perturbative QCD calculations [13] show that $\zeta / s$ vanishes as $\sim \alpha_{s}^{2} / \ln \left(1 / \alpha_{s}\right)$ for massless quarks and, thus, it becomes negligible in comparison to $\eta / s$ in this regime (recent calculations of $\zeta / s$ in a pion gas performed in the regime $T \ll m_{\text {pion }}$ can be found in [14, 15]). Given the multitude of mass states present in QCD at intermediate temperatures in the hadronic phase and the maximal violation of conformal invariance observed in lattice simulations when $T \sim 150-250 \mathrm{MeV}[16$, it has been suggested that $\zeta / s$ may display a peak in the same temperature region [17, 18]. It is not clear at the moment if such a peak exists in QCD, though model calculations have given support to this idea [19, 20] and some of its phenomenological consequences in heavy ion collisions have already been investigated [21 23]. The effects of bulk viscosity in hydrodynamic simulations of the QGP have not been investigated as thoroughly as in the case of shear viscosity. Hydrodynamical calculations that have used nonzero $\zeta / s$ within averaged initial conditions include [24 29] while event-by-event simulations have been studied in [30].

In this paper we study the effects of bulk viscosity on the fluid-dynamical evolution of the QGP and also at freezeout on an event-by-event basis. The effects of bulk viscous pressure on differential collective flow Fourier coefficients $v_{2}-v_{5}$ are computed. Furthermore, a new formula for the bulk viscous corrections in the distribution function at freeze-out is derived starting from the Boltzmann equation for multi-particle hadron species. Our calculations show that differential collective flow Fourier coefficients from $v_{2}$ to $v_{5}$ are enhanced by bulk viscosity, in the range of $p_{T} \sim 1-3 \mathrm{GeV}$ even when $\zeta / s$ is significantly smaller than $1 /(4 \pi)$. Previously, this was only known to occur to $v_{2}$ (see, for instance, [24, 29]). The interplay between shear and bulk viscosities in the evolution and freeze-out of the QGP will be investigated in a forthcoming paper.

The calculations presented in this work were performed using a new relativistic hydrodynamics numerical code called viscous Ultrarelativistic Smoothed Particle hydrodynamics (v-USPhydro). In this code the equations of $2+1$ (i.e, boost invariant) relativistic viscous hydrodynamics are solved using the Smoothed Particle Hydrodynamics (SPH) Lagrangian algorithm originally developed in [31, 32] and later adapted in 33] for applications in heavy ion collisions. $\mathrm{SPH}$ is a mesh free algorithm widely used in several different applications of fluid dynamics that range from cosmology [34] to engineering [35]. Standard grid-based, Eulerian algorithms are known to become very time consuming and eventually run into problems when dealing with fluid dynamical problems involving free surfaces, deformable boundaries, and extremely large deformations [35]. Given the extremely rapid time evolution and the large gradients that appear in event by event simulations of QGP, it is conceivable that mesh free methods such as SPH can be instrumental in solving the equations of relativistic hydrodynamics in heavy ion collision applications. In fact, this algorithm has been the basis for the hydrodynamic part of the well-known NeXSPheRIO code [33, 36 47]. However, an important distinction between v-USPhydro and NeXSPheRIO, besides the assumption of boost invariant dynamics made in v-USPhydro, is that viscous effects in the hydrodynamical evolution and freeze-out are included in v-USPhydro. The details of the code and the tests made to confirm its accuracy are presented in several Appendices.

Definitions: Our metric signature in Minkowski space-time is mostly minus, i.e., +, -, -, - and we use natural units $\hbar=k_{B}=c=1$. We employ Greek indices for the 4-vectors, e.g, the 4-momentum is $p^{\mu}$, space-time coordinates are $x^{\mu}$, the flow field $u^{\mu}$, while we use bold letters for vectors in the transverse plane, e.g., a, and latin indices for the components $a_{i}$. The scalar product among 4 -vectors is denoted as $p \cdot u=p_{\mu} u^{\mu}$ while for spatial vectors we have $\mathbf{a} \cdot \mathbf{b}=a_{x} b_{x}+a_{y} b_{y}$. Throughout this paper we will be using hyperbolic coordinates $x^{\mu}=(\tau, \mathbf{r}, \eta)$ defined by

$$
\begin{aligned}
\tau & =\sqrt{t^{2}-z^{2}} \\
\eta & =\frac{1}{2} \ln \left(\frac{t+z}{t-z}\right) .
\end{aligned}
$$

The metric in hyperbolic coordinates is $g_{\mu \nu}=\left(1,-1,-1,-\tau^{2}\right)$ while the boost invariant configuration for the flow is $u_{\mu}=\left(\sqrt{1+u_{x}^{2}+u_{y}^{2}}, u_{x}, u_{y}, 0\right)$ and, thus, $u \cdot u=1$. 


\section{THE EQUATIONS OF MOTION OF 2+1 VISCOUS RELATIVISTIC HYDRODYNAMICS INCLUDING BULK VISCOSITY}

We assume a vanishing baryon chemical potential and thus investigate only the equations of motion that stem from energy and momentum conservation within a boost invariant setup. The conservation of energy and momentum is given by

$$
\frac{1}{\sqrt{-g}} \partial_{\mu}\left(\sqrt{-g} T^{\mu \nu}\right)+\Gamma_{\lambda \mu}^{\nu} T^{\lambda \mu}=0
$$

where $\sqrt{-g}=\tau$ and the Christoffel symbol is

$$
\Gamma_{\lambda \mu}^{\nu}=\frac{1}{2} g^{\nu \sigma}\left(\partial_{\mu} g_{\sigma \lambda}+\partial_{\lambda} g_{\sigma \mu}-\partial_{\sigma} g_{\mu \lambda}\right) .
$$

The most general expression for the energy-momentum tensor (in the absence of shear viscosity effects) is

$$
T^{\mu \nu}=\varepsilon u^{\mu} u^{\nu}-(p+\Pi) \Delta^{\mu \nu},
$$

where $\Pi$ is the bulk viscous pressure and the spatial projector is $\Delta_{\mu \nu}=g_{\mu \nu}-u_{\mu} u_{\nu}$. Above, we use the Landau definition for the local rest frame, $u_{\nu} T^{\mu \nu}=\varepsilon u^{\mu}$. The remaining dynamical quantities are the energy density $\varepsilon$, the pressure $p$, and the fluid 4 -velocity $u^{\mu}$. Besides energy-momentum conservation, one also needs to specify the differential equation obeyed by $\Pi$. In this paper we employ the simplest second order formulation of the fluid dynamical equations of motion that can be causal and stable

$$
\tau_{\Pi}(D \Pi+\Pi \theta)+\Pi+\zeta \theta=0,
$$

where $D=u^{\mu} \partial_{\mu}$ is the comoving covariant derivative, $\theta=\tau^{-1} \partial_{\mu}\left(\tau u^{\mu}\right)$ is the fluid expansion rate, $\zeta$ is the bulk viscosity, and $\tau_{\Pi}$ is the relaxation time coefficient required to preserve causality (see, e.g., the discussion in [48]). Equation (5) is discussed in detail in [27, 49]. Note that the differential equation for $\Pi$ in (5) includes the nonlinear term $\Pi \theta$. For a full derivation of the equations of motion using the method of moments for the Boltzmann equation including the numerous other terms that enter at that order the reader is referred to [50]. In this paper viscous effects associated with bulk viscosity are encoded in only 2 transport coefficients, but the inclusion of the remaining terms is straightforward and is left for a future work.

Fluid-dynamical evolution can be described using either the Eulerian or the Lagrangian approach. Eulerian methods require the presence of a grid where the hydrodynamical fields are defined while in Lagrangian methods the flow is described following the trajectory of fluid "particles" and one can consistently rephrase the field equations in this language. In cosmology applications, Lagrangian methods have become largely employed because they allow for quick computational times and avoid other limitations that are inherent to grid-based methods. Currently, in heavy ion collisions almost all hydrodynamical codes are written in the Eulerian formulation with the exception of the Lagrangian-based codes in 27, 33, 51]. The SPH formulation of the equations of motion (2 [5) is reviewed in Appendix A. Since the results of this paper are based on a new relativistic fluid dynamics simulation, we describe tests made to the algorithm in Appendix $\mathrm{A}$ and $\mathrm{E}$.

In the following, we shall always assume the Bjorken scaling solution for the longitudinal direction, in which the component of the velocity field in the longitudinal direction (in hyperbolic coordinates) is set to zero, i.e., $u^{\eta}=0$.

\section{PARAMETERS OF THE MODEL}

As in any hydrodynamical modeling of heavy ion collisions, there are a number of free parameters that must be fixed. The initial time to start the fluid-dynamical evolution is fixed to be $\tau_{0}=1 \mathrm{fm}$. The initial conditions for the hydrodynamic simulations are taken from a Monte Carlo Glauber code [52] in which the initial energy density is given in the form

$$
\varepsilon(\mathbf{r})=c n_{\text {coll }}(\mathbf{r})
$$

where $n_{\text {coll }}$ is the number density of binary collisions in the event and the constant $c$ is fixed to describe the final multiplicities observed experimentally. The centrality classes we used are defined in terms of the number of participant nucleons and are in agreement with standard results from other Monte Carlo Glauber simulations 53]. We assumed that for $\sqrt{s}=200 \mathrm{GeV}$ RHIC's most central collisions at mid-rapidity there are about $300 \pi^{+}$'s [54] and, since we 

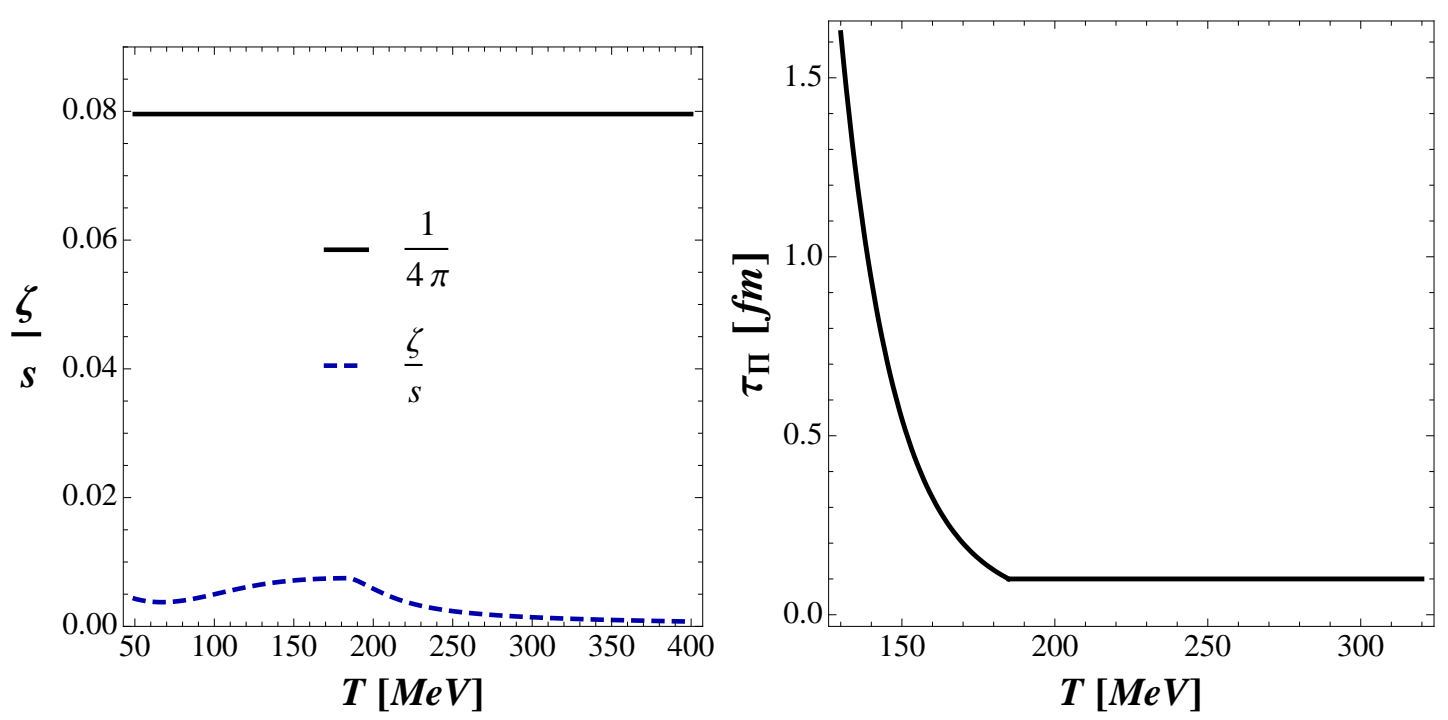

FIG. 1. The temperature dependence of the transport coefficients $\zeta / s$ and $\tau_{\Pi}$ (see Eqs. (77) and (8), respectively).

assume that freeze-out occurs at $T=150 \mathrm{MeV}$, we estimate that about $41 \%$ of pions would come from direct thermal pions [55]. Thus, the constant $c$ was adjusted for the ideal case using an average 0-5\% most central RHIC event so that we obtain $123 \pi^{+}$'s when $T_{F O}=150 \mathrm{MeV}$. Note that when bulk viscosity is included the total entropy of the system increases in time and, in order to keep the total number of pions at freeze-out the same as before, one has to slightly reduce $c$. Here, we assume that $\Pi$ and the spatial components of $u^{\mu}$ vanish at $\tau_{0}$.

Initial conditions that include the effects of gluon saturation, such as those in [52, 56, 57], can also be studied using v-USPhydro. However, this type of initial conditions displays structure on smaller length scales than that of the usual Glauber initial conditions and, thus, smaller values of the smoothing parameter $h$ (more on that below) must be used to systematically investigate the difference created in the flow harmonics due to gluon saturation effects at early times.

We use the lattice-based equation of state from [58] with chemical equilibrium. We have tested the dependence of our results with the choice for the equation of state by using the equation of state from Fodor et al. [16] which, however, led to no noticeable difference in the flow harmonics. The results shown in this paper were computed using EOS S95n-v1 [58] (for $T<50 \mathrm{MeV}$ this EOS is matched to that of a massive gas of pions).

The bulk viscosity coefficient is parametrized in the following way

$$
\frac{\zeta}{s}=\frac{1}{8 \pi}\left(\frac{1}{3}-c_{s}^{2}\right),
$$

where $s$ is the entropy density and $c_{s}$ is the speed of sound. As one can see in Fig. $1, \zeta / s$ is significantly smaller than the standard value of $\eta / s \sim 1 /(4 \pi)$. Additionally, because of the dip in the speed of sound, there is a peak in $\zeta / s$ between $150-200 \mathrm{MeV}$. This formula for the bulk viscosity is inspired by Buchel's formula obtained within the gauge/gravity duality [59]. Calculations performed in [13] showed that at sufficiently large $T$ in the QGP phase the bulk viscosity in QCD follows the relation $\zeta / \eta \sim 15\left(1 / 3-c_{s}^{2}\right)^{2}$. The specific functional form of $\zeta / s$ does not make a significant difference in our results as long as the overall magnitude is the same.

The temperature dependence of the relaxation time, $\tau_{\Pi}$, is described by the formula found in [60]

$$
\tau_{\Pi}=9 \frac{\zeta}{\varepsilon-3 p}
$$

and shown in Fig. 1. Clearly, other choices for $\tau_{\Pi}$ are possible but, for simplicity, in this paper we fix it as above. We chose a time step $d \tau=0.1 \mathrm{fm}$ in our numerical simulations and $\tau_{\Pi}$ is set to never be smaller than $d \tau$ (this is why $\tau_{\Pi}$ plateaus when $T>0.2 \mathrm{GeV}$ ). Also, the time step is considerably smaller than $\tau_{0}$, as required to resolve the gradients in the longitudinal direction.

We set the isothermal freeze-out temperature in the Cooper-Frye procedure [61] to be $T_{F O}=150 \mathrm{MeV}$. The Cooper-Frye freeze-out method is written in the SPH language in Appendix B Lower freeze-out temperatures have a strong effect on the viscous corrections to the Cooper-Frye freeze-out, as it will be explained in the next section. On 
the other hand, higher freeze-out temperatures can run into issues because of the effects coming from the production of heavy resonances that could contribute strongly to the flow harmonics [62]. In this paper all flow harmonics are shown for direct thermal $\pi^{+}$, without the inclusion of a hadronic afterburner or particle decays. Thus, the purpose of the present study is to understand the qualitative effects of bulk viscosity on the flow harmonics event by event. In the future, we will include particle decays (and possibly hadronic afterburner effects) into v-USPhdro so that the results of our calculations can be more directly compared to experimental results.

As explained in detail in Appendix A, the Lagrangian formalism we used includes a parameter, $h$, known as the SPH length scale. The $h$ parameter essentially works as a smoothing parameter such that a larger $h$ smoothes out initial conditions to the point that fluctuations are minimized whereas a too small $h$ requires a very large number of SPH particles so that it overwhelms standard computational times. Thus, one needs to choose a value of $h$ that is small enough to preserve as much of the structure in the initial conditions as possible but that also allows for a realistic running time. We found that $h=0.3 \mathrm{fm}$ and $N_{S P H}=27000$ allow for relatively quick running times $(\sim 10$ minutes per hydro event in a single standard machine) while still preserving the structure seen in the Monte Carlo Glauber initial fluctuations for both centrality classes studied in this paper. If one increases $h$ beyond that value the initial fluctuations are smoothed out and we see $v_{2}$ increase by about $5 \%$ at high $p_{T}$ when one uses $h=0.5 \mathrm{fm}$. For further details and discussion see Appendix E, Conservation of energy and momentum in our simulations hold up to $0.01 \%$.

\section{BULK VISCOSITY CONTRIBUTION TO THE MULTI-HADRON DISTRIBUTION FUNCTION}

\section{A. Method of Moments}

In order to compute the particle distribution at freeze-out, one needs to evaluate the non-equilibrium distribution function for each hadron species on the freeze-out hypersurface. In general, the distribution function for each hadron species is $f_{\mathbf{k}}^{(i)}=f_{0 \mathbf{k}}^{(i)}+\delta f_{\mathbf{k}}^{(i)}$, where $f_{0 \mathbf{k}}^{(i)}=\left[\exp \left(\beta_{0} E_{i \mathbf{k}}-\alpha_{0}\right)+a\right]^{-1}$ is the local equilibrium distribution function and $\delta f_{\mathbf{k}}^{(i)}$ is the corresponding non-equilibrium part. In this work, we use the method of Moments, as developed in Refs. [50, 63], to compute the non-equilibrium contribution $\delta f_{\mathbf{k}}^{(i)}$ associated with bulk viscosity effects to the momentum distribution function of a hadronic mixture.

First, we factorize $\delta f_{\mathbf{k}}^{(i)}$ in the following way

$$
\delta f_{\mathbf{k}}^{(i)}=f_{0 \mathbf{k}}^{(i)} \tilde{f}_{0 \mathbf{k}}^{(i)} \phi_{\mathbf{k}}^{(i)}
$$

where $\tilde{f}_{0 \mathbf{k}}^{(i)}=1+a f_{0 \mathbf{k}}^{(i)}\left(a=-1 / 1\right.$ for fermions/bosons), and $\phi_{\mathbf{k}}^{(i)}$ is an out-of-equilibrium contribution. Next, $\phi_{\mathbf{k}}^{(i)}$ is expanded in terms of its moments using a complete and orthogonal basis constructed from particle four-momentum, $k_{i}^{\mu}$, and fluid four-velocity, $u^{\mu}$. As done in Refs. [50, 63], we use an expansion basis with two basic ingredients: the irreducible tensors

$$
1, k_{i}^{\langle\mu\rangle}, k_{i}^{\langle\mu} k_{i}^{\nu\rangle}, k_{i}^{\langle\mu} k_{i}^{\nu} k_{i}^{\lambda\rangle}, \cdots,
$$

analogous to the well-known set of spherical harmonics and constructed by the symmetrized traceless projection of $k_{i}^{\mu_{1}} \cdots k_{i}^{\mu_{m}}$, i.e., $k_{i}^{\left\langle\mu_{1}\right.} \cdots k_{i}^{\left.\mu_{m}\right\rangle} \equiv \Delta_{\nu_{1} \cdots \nu_{m}}^{\mu_{1} \cdots \mu_{m}} k_{i}^{\nu_{1}} \cdots k_{i}^{\nu_{m}}$, and the orthonormal polynomials

$$
P_{i \mathbf{k}}^{(n \ell)}=\sum_{r=0}^{n} a_{n r}^{(\ell) i}\left(u \cdot k_{i}\right)^{r}
$$

which are equivalent to the associated Laguerre polynomials in the limit of massless, classical particles.

Then, the momentum distribution function of the $i$-th particle species becomes,

$$
f_{\mathbf{k}}^{(i)}=f_{0 \mathbf{k}}^{(i)}+f_{0 \mathbf{k}}^{(i)} \tilde{f}_{0 \mathbf{k}}^{(i)} \sum_{\ell=0}^{\infty} \sum_{n=0}^{\infty} \mathcal{H}_{i \mathbf{k}}^{(n \ell)} \rho_{i, n}^{\mu_{1} \cdots \mu_{\ell}} k_{i, \mu_{1}} \cdots k_{i, \mu_{\ell}}
$$

where we introduced the energy-dependent coefficients, $\mathcal{H}_{i \mathbf{p}}^{(n \ell)} \equiv\left[N_{i}^{(\ell)} / \ell !\right] \sum_{m=n}^{\infty} a_{m n}^{(\ell) i} P_{i \mathbf{k}}^{(m \ell)}\left(u \cdot k_{i}\right)$ (see the details in [50]). The fields $\rho_{i, n}^{\mu_{1} \cdots \mu_{\ell}}$ can be exactly determined using the orthogonality relations satisfied by the expansion basis and can be shown to correspond to irreducible moments of $\delta f_{\mathbf{k}}^{(i)}$,

$$
\rho_{i, r}^{\mu_{1} \ldots \mu_{\ell}} \equiv\left\langle E_{i \mathbf{k}}^{r} k_{i}^{\left\langle\mu_{1}\right.} \ldots k_{i}^{\left.\mu_{\ell}\right\rangle}\right\rangle_{\delta}, \quad\langle\ldots\rangle_{\delta}=\int d K_{i}(\ldots) \delta f_{\mathbf{k}}^{(i)},
$$


where $g_{i}$ is the degeneracy factor of the $i$-th hadron species and $d K_{i}=g_{i} d^{3} \mathbf{k} /\left[(2 \pi)^{3} k_{i}^{0}\right]$. As long as this basis is complete, the above expansion fully describes $f_{\mathbf{k}}^{(i)}$, no matter how far from equilibrium the system is.

Here, we are interested only on the effects arising from the bulk viscous pressure. For this case, it is enough to consider only the $\ell=0$ (scalars) terms in the expansion above, i.e., neglect all irreducible first-rank tensors, e.g. heat flow, second-rank tensors, e.g. shear-stress tensor, and tensors with rank higher than two (that never appear in fluid dynamics). The next approximation is the truncation of the expansion in momentum space, keeping only the terms corresponding to $n=0,1,2$ (for $\ell=0$ ). Then, we obtain (for classical particles)

$$
\begin{aligned}
f_{\mathbf{k}}^{(i)}= & f_{0 \mathbf{k}}^{(i)}+\delta f_{\mathbf{k}}^{(i)} \\
\delta f_{\mathbf{k}}^{(i)}= & \frac{f_{0 \mathbf{k}}^{i}}{J_{00}^{i}}\left\{\left[1+a_{10}^{(0) i} a_{10}^{(0) i}+a_{20}^{(0) i} a_{20}^{(0) i}+\left(a_{10}^{(0) i} a_{11}^{(0) i}+a_{20}^{(0) i} a_{21}^{(0) i}\right) u \cdot k_{i}+a_{20}^{(0) i} a_{22}^{(0) i}\left(u \cdot k_{i}\right)^{2}\right] \rho_{i(0)}\right. \\
& \left.+a_{22}^{(0) i}\left[a_{20}^{(0) i}+a_{21}^{(0) i} u \cdot k_{i}+a_{22}^{(0) i}\left(u \cdot k_{i}\right)^{2}\right] \rho_{i(2)}\right\} .
\end{aligned}
$$

The coefficients $J_{m n}^{i}$ and $a_{m n}^{(0) i}$ are thermodynamic quantities that appear in the definition of the orthogonal polynomicals $P_{i \mathbf{k}}^{(n \ell)}$. These functions are defined in Appendix D. Note that $m_{i}^{2} \rho_{i, 0}=\rho_{i, 2}-3 \Pi_{i}$, with $\Pi_{i}$ being the bulk viscous pressure of the $i$-th particle species, respectively. Likewise, $\varepsilon_{i}$, and $p_{i}$ are the energy density and thermodynamic pressure of the $i$-th particle species, respectively.

In order to apply this expression to describe freeze-out, further approximations are required. This happens because in fluid dynamics one evolves the total bulk viscous pressure of the system $\left(\Pi=\sum_{i} \Pi_{i}\right)$ and it is not possible to know, just from fluid dynamics itself, how these quantities are distributed among the individual bulk viscous pressure of each hadron species $\left(\Pi_{i}\right)$. We remark that $\Pi=-\sum_{i}\left(m_{i}^{2} / 3\right) \rho_{i, 0}$ and $\sum_{i} \rho_{i, 2}=0$, the second relation arising from the Landau matching condition. Therefore, we need to relate the scalar moments $\rho_{i, 0}, \rho_{i, 1}$, and $\rho_{i, 2}$ to the fluid-dynamical variables $\Pi$.

In this work, we assume that the system is close to the Navier-Stokes limit. Even though this assumption does not happen in practice, we consider it good enough to provide a rough estimate for the non-equilibrium distribution function. In this case,

$$
\Pi=-\zeta \theta, \rho_{i, m}=-\alpha_{i, m} \theta \Longrightarrow \rho_{i, m}=\frac{\alpha_{i, m}}{\zeta} \Pi
$$

Then

$$
\begin{aligned}
& \delta f_{\mathbf{k}}^{(i)}=\frac{f_{0 \mathbf{k}}^{i}}{J_{00}^{i}} \Pi\left\{\left[1+a_{10}^{(0) i} a_{10}^{(0) i}+a_{20}^{(0) i} a_{20}^{(0) i}+\left(a_{10}^{(0) i} a_{11}^{(0) i}+a_{20}^{(0) i} a_{21}^{(0) i}\right) u \cdot k_{i}+a_{20}^{(0) i} a_{22}^{(0) i}\left(u \cdot k_{i}\right)^{2}\right] \frac{\alpha_{i, 0}}{\zeta}\right. \\
& \left.+a_{22}^{(0) i}\left[a_{20}^{(0) i}+a_{21}^{(0) i} u \cdot k_{i}+a_{22}^{(0) i}\left(u \cdot k_{i}\right)^{2}\right] \frac{\alpha_{i, 2}}{\zeta}\right\} \\
& =f_{0 \mathbf{k}}^{i} \Pi\left[B_{0}^{(i)}+D_{0}^{(i)} u \cdot k_{i}+E_{0}^{(i)}\left(u \cdot k_{i}\right)^{2}\right] \text {. }
\end{aligned}
$$

The coefficients $B_{0}^{(i)}, D_{0}^{(i)}$, and $E_{0}^{(i)}$ are defined as implied. In general, while these coefficients depend on the freeze-out temperature and the particle's mass and degeneracy, they cannot be simply expressed in terms of thermodynamical quantities such as energy density per particle and etc.

Note that the dependence of $\delta f^{(i)}$ with the particle's 4-momentum is qualitatively different than that commonly used for shear viscous effects where $\delta f_{\text {shear }} \sim \pi_{\mu \nu} k_{i}^{\mu} k_{i}^{\nu} /\left(\varepsilon_{i}+p_{i}\right) / T^{2}$, where $\pi^{\mu \nu}$ is the shear tensor [63]. Taking the equivalent Navier-Stokes limit for the shear correction, one can see the relevant quantity in this case is $\eta / s$. Thus, if $\eta / s$ is sufficiently small, the $\delta f$ expansion may still be well defined for intermediate values of $p_{T}$. However, for bulk viscosity, even in the Navier-Stokes limit the relevant quantity in the $\delta f$ series is not $\zeta / s$. Rather, one can see that at low momenta the relevant quantity is $\sim \zeta \theta B_{0}$ while as one increases the momentum the important quantity becomes $\sim E_{0}\left(u \cdot k_{i}\right)^{2} \zeta \theta$. Therefore, a relatively small $\zeta / s$ (in comparison to $\eta / s$, for example) does not necessarily mean that the actual values that matter in the viscous contribution to freeze-out are going to be small.

\section{B. Simple Model of Hadrons}

In order to compute the coefficients $\alpha_{i, m}$ appearing in $\delta f_{\mathbf{k}}^{(i)}$ we must provide a set of hadronic cross sections. In this work, we estimate these coefficients using a simple hadronic model in which all hadrons have the same constant cross section. Note that in this case the ratios $\alpha_{i, 0} / \zeta$ and $\alpha_{i, 2} / \zeta$ are actually independent of the value of cross section 
chosen. We consider only elastic collisions between the hadrons and include all hadrons up to a mass of 1.2 GeV (heavier hadrons are not included due to the exceedingly large computational cost).

Since we consider only elastic collisions among the hadrons, the particle number of individual species is conserved and the moments $\rho_{i, 1}$ vanish, $\rho_{i, 1}=0$. In this case, the coefficients $B_{0}, D_{0}$, and $E_{0}$ for pions with freeze-out temperature $T_{F O}=150 \mathrm{MeV}$ are

$$
\begin{aligned}
& B_{0}^{(\pi)}=-65.85 \mathrm{fm}^{4} \\
& D_{0}^{(\pi)}=171.27 \mathrm{fm}^{4} / \mathrm{GeV}, \\
& E_{0}^{(\pi)}=-63.05 \mathrm{fm}^{4} / \mathrm{GeV}^{2}
\end{aligned}
$$

and the non-equilibrium correction in the distribution function for pions is

$$
\delta f_{\mathbf{k}}^{(\pi)}=f_{0 \mathbf{k}}^{\pi} \Pi\left[B_{0}^{(\pi)}+D_{0}^{(\pi)} u \cdot k_{\pi}+E_{0}^{(\pi)}\left(u \cdot k_{\pi}\right)^{2}\right]
$$

For freeze-out temperatures lower than $150 \mathrm{MeV}$, the $\delta f$ contribution to the distribution function can become comparable to the ideal distribution $f_{0}$, which makes a perturbative analysis of the viscous effects at freeze-out untrustworthy. Thus, we used $T_{F O}=150 \mathrm{MeV}$ for the calculations in this paper.

The Moments method [50, 63] can be used to derive a relativistic dissipative fluid-dynamical theory from kinetic theory which provides a good description of all dissipative phenomena. This has been explicitly demonstrated in [64, 65] where calculations performed within this theory were shown to match the corresponding numerical solutions of the relativistic Boltzmann equation. However, in order to estimate the systematic uncertainties in our calculation due to the approximations performed in this section in the determination of $\delta f$, we also consider two other options. The first one corresponds to the result derived by Monnai and Hirano in [24] using an implementation of Grad's 14-moment method for multi-particle species. The other formula for $\delta f$ was obtained by Dusling and Schäfer in [29] using the relaxation time approximation and the assumption that the deviation from equilibrium for each hadron species comes the near-zero mode similar to that found in scalar field theory [15]. The formulas for $\delta f$ computed in these works can be put in the form (18) with the coefficients:

$$
\begin{aligned}
& B_{0}^{(\pi)}=-0.69 \mathrm{fm}^{4} \\
& D_{0}^{(\pi)}=-38.96 \mathrm{fm}^{4} / \mathrm{GeV}, \\
& E_{0}^{(\pi)}=49.69 \mathrm{fm}^{4} / \mathrm{GeV}^{2}
\end{aligned}
$$

for [24] and

$$
\begin{aligned}
& B_{0}^{(\pi)}=-71.96 \mathrm{fm}^{4} \\
& D_{0}^{(\pi)}=121.50 \mathrm{fm}^{4} / \mathrm{GeV} \\
& E_{0}^{(\pi)}=0
\end{aligned}
$$

for [29].

In Fig. 2 the variation of $v_{2}\left(p_{T}\right)$ with the different Ansätze for the $\delta f$ contributions from the bulk viscosity is shown. The results are for mid-rapidity RHIC's $\sqrt{200} \mathrm{GeV} 20-30 \%$ most central collisions in the case where the initial condition corresponds to a single average (over 150 events) Glauber initial condition. The ideal case is the solid black line, our result for $v_{2}$ computed using the $\delta f$ obtained via the Moments method is the long dashed black line, results for the $\delta f$ described in [29] is the short dashed red curve, while the short and long dashed brown curve is the result computed using the $\delta f$ described in [24]. One can see that all the different approaches lead to an enhancement of $v_{2}\left(p_{T}\right)$ when $p_{T} \sim 1-3 \mathrm{GeV}$ in comparison to the ideal fluid case. However, note that $v_{2}\left(p_{T}\right)$ is more well behaved in the intermediate $1-3 \mathrm{GeV}$ range when the result for $\delta f$ computed within the Moments method developed in [50, 63] is used in comparison to the expressions obtained in [24] and [29]. In the next section, the newly developed formula in Eq. (18) will be used to study the effects of bulk viscosity on the collective flow harmonic coefficients.

\section{RESULTS FOR THE COLLECTIVE FLOW COEFFICIENTS}

In this section we present our results for the effects of bulk viscosity on the collective flow coefficients associated with direct, thermal $\pi^{+}$. The flow coefficients were computed event by event using the event plane method [66] described for completeness in Appendix C] In the following all results were computed for $p_{T}=0-3 \mathrm{GeV}$ for RHIC's $\sqrt{s}=200$ 


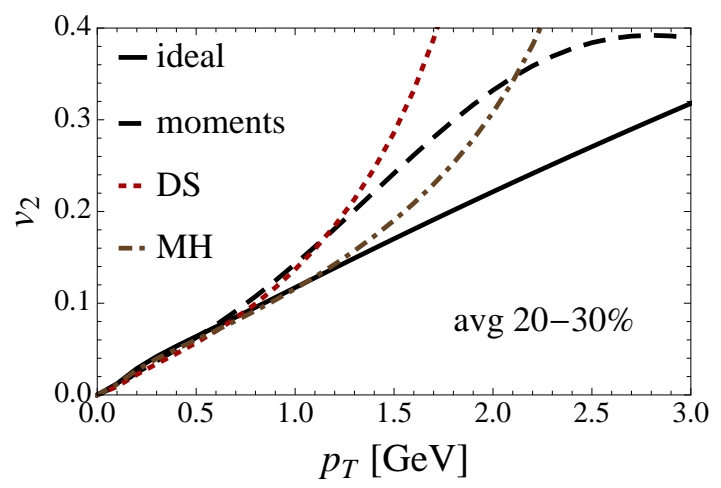

FIG. 2. Dependence of the direct $\pi^{+}$differential elliptic flow on the specific formula for the viscous $\delta f$ contribution from the bulk viscosity that enters in the Cooper-Frye freeze-out. The results are for RHIC's $\sqrt{200}$ GeV $20-30 \%$ most central collisions in the case where the initial condition corresponds to a single average Glauber initial condition. The ideal case is the solid black line, our result for $v_{2}$ computed using the $\delta f$ obtained via the Moments method is the long dashed black line, results for the $\delta f$ described in [29] is the short dashed red curve, while the short and long dashed brown curve is the result computed using the $\delta f$ described in 24$]$.

$\mathrm{GeV}$ most central collisions $(0-5 \%)$ and peripheral collisions $(20-30 \%)$. For each centrality class we have considered 150 events. In Fig. 3 we show a comparison of the effects of bulk viscosity in event-by-event Glauber initial conditions vs. averaged Glauber initial conditions for the $0-5 \%$ most central collisions. For event by event initial conditions, the black solid line shows $v_{2}\left(p_{T}\right)$ for an ideal fluid while the long dashed black line shows the effect of bulk viscosity from Eq. (18) both in the hydrodynamical evolution and freeze-out. For a single averaged Glauber simulation, the red dashed dotted line shows $v_{2}\left(p_{T}\right)$ in the case of an ideal fluid while the short dashed red line shows the effect of bulk viscosity both in the hydrodynamical evolution and freeze-out. As one can see in Fig. 3 bulk viscosity enhances $v_{2}\left(p_{T}\right)$ when $p_{T} \sim 1-3 \mathrm{GeV}$ with respect to the ideal fluid result and this enhancement is actually more pronounced in event by event simulations. We shall see in the following that this enhancement is also present for higher flow harmonics.

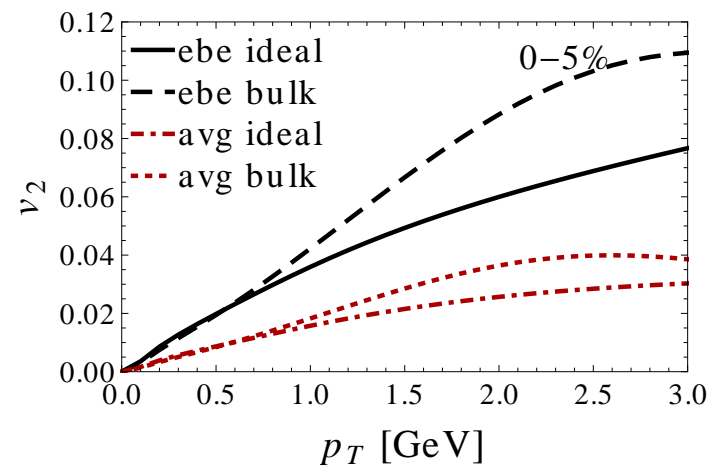

FIG. 3. Comparison of the effects of bulk viscosity in event-by-event Glauber initial conditions vs. averaged Glauber initial conditions for RHIC's $\sqrt{s}=200 \mathrm{GeV} 0-5 \%$ most central collisions. We have considered 150 events. For event by event simulations, the black solid line shows $v_{2}\left(p_{T}\right)$ for an ideal fluid while the long dashed black line shows the effect of bulk viscosity both in the hydrodynamical evolution and freeze-out. In the case of a single averaged Glauber simulation, the red dashed dotted line shows $v_{2}\left(p_{T}\right)$ for an ideal fluid while the short dashed red line shows the effect of bulk viscosity both in the hydrodynamical evolution and freeze-out.

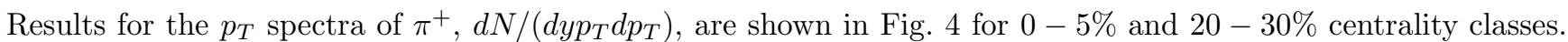
Effects from particle decays are not included in any of the plots shown in this paper. One can see that bulk viscosity steepens the spectra in both centrality classes compared to the ideal case, as can be guessed from Eq. (18). This is responsible for the enhancement in $v_{2}\left(p_{T}\right)$ at high $p_{T}$, as remarked by [24] and [29]. Note that when the $\delta f$ correction is not included in the Cooper-Frye procedure (i.e., $\delta f=0$ ), the effect of bulk viscosity on spectra is very small in both centrality classes.

In Fig. 5 the flow harmonics from $v_{2}$ to $v_{5}$ are shown for our two centrality classes for event-by-event Glauber initial conditions. In all the different plots in Fig. 5. solid lines correspond to the ideal fluid dynamics solution, whereas short 

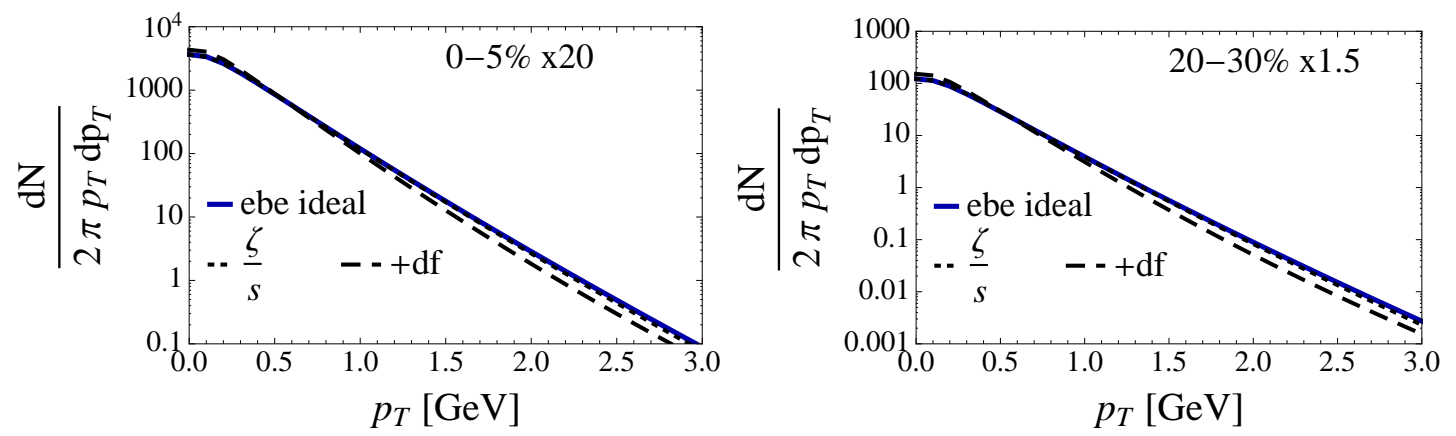

FIG. 4. The $\pi^{+}$spectra $d N /\left(2 \pi p_{T} d p_{T}\right)$ for $0-5 \%$ and $20-30 \%$ centrality classes. The ideal fluid case is shown in a solid blue line, the result in the case where effects of bulk viscosity are included only on the hydrodynamical evolution but not on the freeze-out is shown by the short dashed black line while the long dashed black curve includes bulk effects on both the hydro evolution and freeze-out.

dashed curves include the effects of bulk viscosity only on the hydrodynamical evolution (i.e., $\delta f=0$ at freeze-out) and the long dashed curve takes into account the effects of bulk viscosity both in the hydro evolution and at freeze-out.

Note that, similarly to what is generally seen in the case of shear viscosity [67], when the contribution from $\delta f$ is not included in the Cooper-Frye procedure the overall effect of bulk viscosity on the differential flow anisotropies is small, with basically no deviation from the ideal fluid result. On the other hand, when one considers the additional non-equilibrium correction $\delta f$ there is a universal enhancement in the $v_{n}$ 's regardless of centrality class, mostly above $p_{T}=1 \mathrm{GeV}$. The effect is most significant in non-central collisions. Thus, bulk viscosity affects higher order flow harmonics in the opposite way that shear viscosity does. In fact, while shear viscosity suppresses $v_{n}\left(p_{T}\right)$, bulk viscosity actually enhances it. One could expect that in the case where both shear and bulk viscosity are included in event by event simulations there could be some competition between the two effects. This interesting question is left for a future study.

In Fig. [6 we show our results for the integrated $v_{n}$ coefficients for the two centrality classes. The plot shows $v_{n}$ divided by the corresponding ideal fluid result as a function of the mode number $n$. For the bulk viscosity and relaxation time coefficients used in this work, we found that the integrated $v_{n}$ 's computed in the viscous fluid are only slightly lower than those found for the ideal fluid, for both centrality classes. This indicates that the value of $\zeta / s$ chosen in this work is small and does not affect the fluid 4-velocity and temperature by much.

On the other hand, as one increases the bulk viscosity coefficient the viscous effects on the integrated $v_{n}$ can become large (when compared to the ideal fluid solution, $v_{n}^{\text {ideal }}$ ). In Fig. 7 we show the ratio $v_{n} / v_{n}^{\text {ideal }}$ computed for several values of $\zeta / s$. This simulation was performed for an initial condition constructed from an average over $150 \mathrm{MC}$ Glauber events taken from the $20-30 \%$ centrality class. Note that, even though this initial condition is considerably smoother than the usual MC Glauber one, it still has a finite $v_{3}$ and $v_{5}$. Also, the integrated $v_{n}$ 's showed in Fig. 7 were computed without the $\delta f$ correction. We remark that the $\delta f$ correction has a very small effect on integrated flow harmonics and should not contribute much for this plot. One can see that, when the $\zeta / s$ taken from Eq. (7) is multiplied by 8 (leaving it with approximately the same magnitude as the shear viscosity coefficient, $\zeta / s \sim 0.08$ ) the flow harmonics are considerably reduced by bulk viscosity. If we multiply it by 16 , the effect is even greater. This result indicates that, if the order of magnitude of the bulk viscosity is close to the one expected for the shear viscosity (as may happen in the hadronic phase), it is not a good approximation to neglect it.

We did not compute $v_{n}\left(p_{T}\right)$ for such larger values of $\zeta / s$ because the $\delta f$ correction (for any of the $\delta f$ 's discussed in this paper) becomes too large and, consequently, renders the resulting calculation meaningless. This indicates several possibilities: 1) the bulk viscous $\delta f$ 's computed so far in the field are still not precise enough 2) the bulk viscosity coefficient is actually very small, and/or 3 ) the $\delta f$ originating from shear viscosity will cancel the one from bulk, allowing for larger values of bulk viscosity to be used even for the currently existing $\delta f$ 's. From the results of this paper, we are not able to state which of the above is actually true.

\section{CONCLUSIONS}

In this paper we used the newly developed, Lagrangian $2+1$ viscous hydrodynamic code v-USPhydro to study the effects of bulk viscosity on the collective flow harmonics observed in ultrarelativistic heavy ion collisions. We found that flow harmonics can be significantly affected by bulk viscosity effects even in the case where the maximum of the 

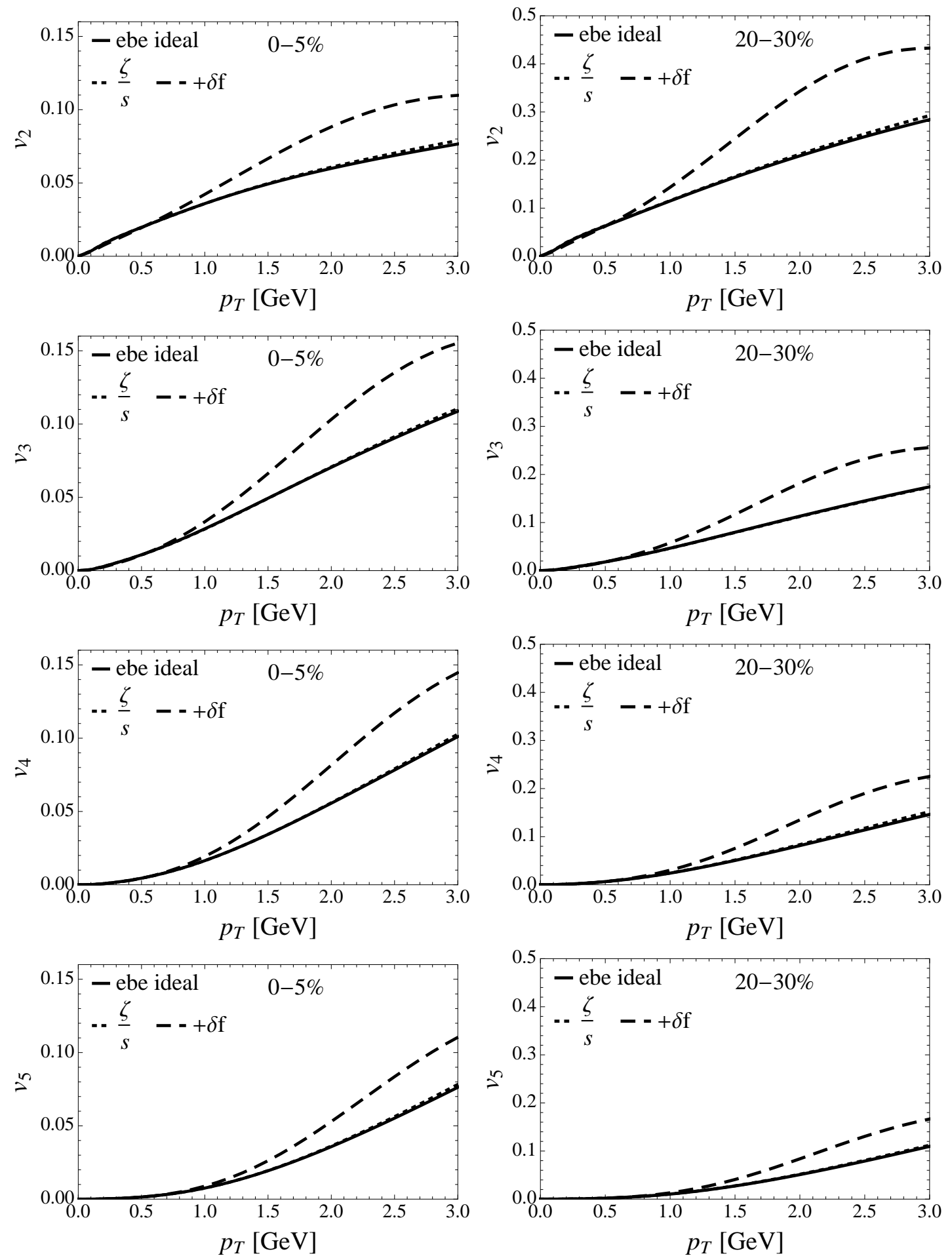

FIG. 5. Results for the bulk viscosity $\zeta / s$ shown for most central collisions $(0-5 \%)$ and non-central collisions $(20-30 \%)$ computed using event-by-event simulations. The solid lines corresponds to the ideal fluid result, the short dashed lines include bulk viscosity only on the hydrodynamical evolution but not at freeze-out while the long dashed lines include bulk viscosity effects both on the hydro evolution and at freeze-out. 

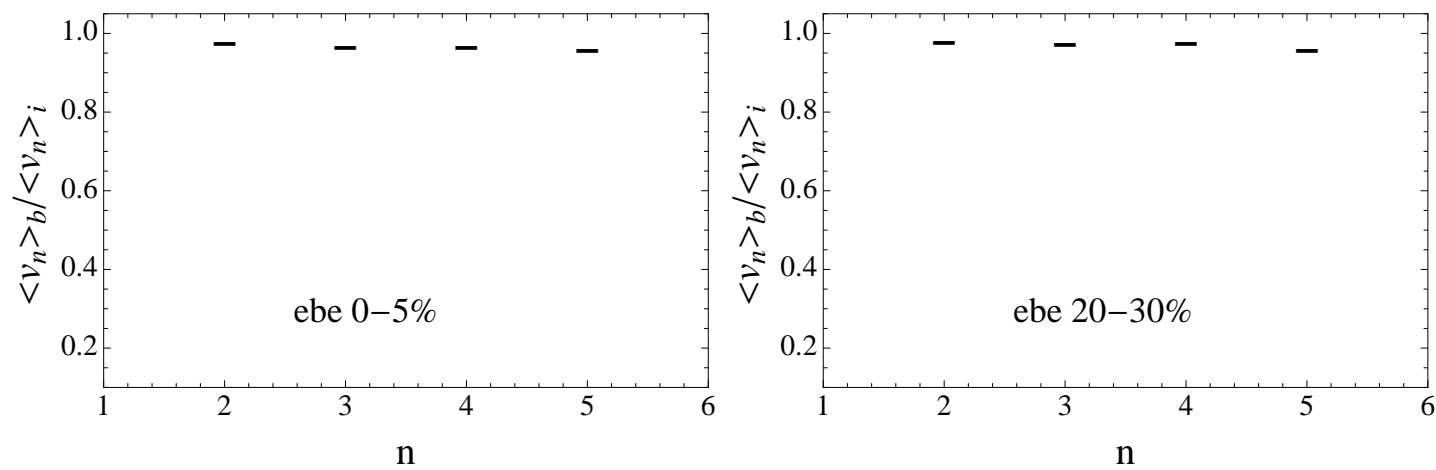

FIG. 6. Ratio between the integrated flow coefficients $v_{n}$ 's including bulk viscosity effects and the corresponding ideal fluid results, computed in event by event simulations, as a function of $n$ for most central collisions $(0-5 \%)$ and peripheral collisions $(20-30 \%)$.

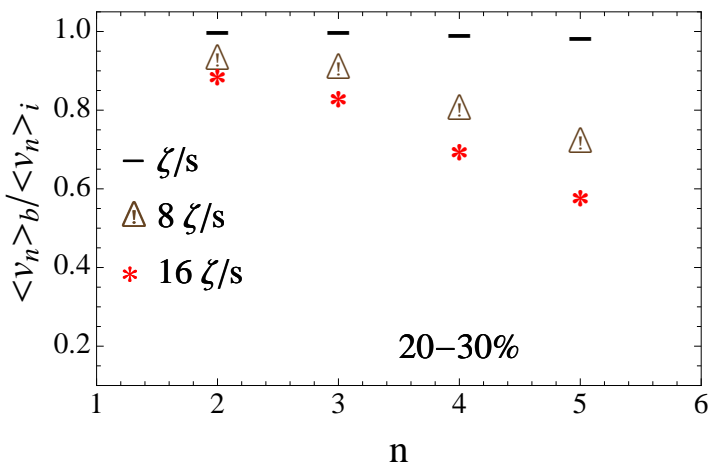

FIG. 7. Ratio between the integrated flow coefficients $v_{n}$ 's including bulk viscosity effects and the corresponding ideal fluid results as a function of $n$. This calculation was performed using an initial condition averaged over $150 \mathrm{MC}$ Glauber events of the $20-30 \%$ centrality class.

temperature dependent $\zeta / s$ is nearly an order of magnitude smaller than the standard $\eta / s=1 /(4 \pi)$ value commonly used in hydrodynamical simulations including only shear viscosity. The inclusion of bulk viscous effects at freeze-out for a system of multi-hadron species was computed using the Moments method [50, 63], which led to a consistent non-equilibrium correction to the distribution function of pions that remains well behaved when $p_{T}=0-3 \mathrm{GeV}$. It would be interesting to investigate how the inclusion of heavier hadrons and experimentally measured hadron cross sections affects the coefficients in $\delta f$ of each hadron species.

We performed event by event simulations (using Monte-Carlo Glauber initial conditions) that allowed us to study for the first time the effects of bulk viscosity on collective flow harmonics of higher order. We have found that bulk viscosity enhances the differential flow coefficients $v_{n}\left(p_{T}\right)$, for $n=2,3,4$, and 5 , with respect to their ideal fluid values when $p_{T} \sim 1-3 \mathrm{GeV}$. This shows that bulk viscosity affects differential flow anisotropies in the opposite way than that found in the case of shear viscosity, which is known to lead to an overall suppression of $v_{n}\left(p_{T}\right)$ in the same intermediate $p_{T}$ range. Thus, our results indicate that a realistic description of the QGP hydrodynamical evolution should include (preferably temperature dependent) shear and bulk viscosities in order to correctly describe the suppression of differential flow harmonics within relativistic heavy ion collisions. The bulk viscosity driven enhancement of $v_{n}\left(p_{T}\right)$ found in this paper also opens up the interesting possibility that bulk and shear viscosity effects may actually compete in the suppression of flow anisotropies in the viscous QGP.

It should be noted that all the $\delta f$ 's used in this work imply that even a small bulk viscosity can have a large effect on differential flow harmonics. This happens for values of bulk viscosity that do not even affect the fluid-dynamical evolution of the plasma. For values of bulk viscosity of $\zeta / s \sim 0.08$, which actually have a considerable effect on the fluid-dynamical evolution of the system, the $\delta f$ correction arising from bulk terms becomes too large, making it physically meaningless to apply it to compute $p_{T}$-differential observables. Therefore, it is important to verify how precise the current $\delta f$ 's in the field actually are. For the case of the moment expansion this can be verified by checking the convergence of the series. It is possible that the truncation employed in this work is still far from the converged 
solution, but this can only be confirmed in a future work.

J. Noronha-Hostler, R. P. G. Andrade, J. Noronha, and F. Grassi acknowledge Fundação de Amparo à Pesquisa do Estado de São Paulo (FAPESP) and Conselho Nacional de Desenvolvimento Científico e Tecnológico (CNPq) for financial support. G. S. Denicol is supported by the Natural Sciences and Engineering Research Council of Canada. The authors thank T. Kodama, Y. Hama, and F. Gardim for discussions on the SPH approach to relativistic fluid dynamics and A. Dumitru for providing the Monte Carlo Glauber model. 


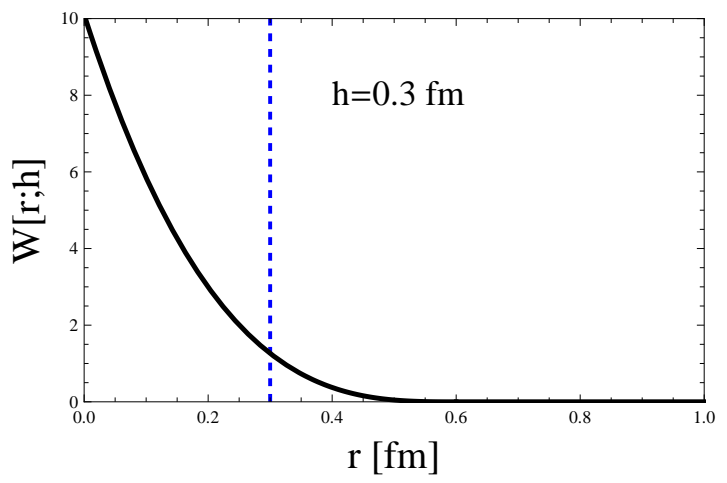

FIG. 8. Kernel function $W[\mathbf{r} ; h]$ as a solid black curve for $h=0.3 \mathrm{fm}$ (denoted by the vertical dashed blue line) used in this paper.

\section{Appendix A: Relativistic Fluid Dynamic Equations in the SPH Formalism}

In the SPH approach one introduces a conserved reference density current $J^{\mu}=\sigma u^{\mu}$ where $\sigma$ is the local density of a fluid element in its rest frame. As the fluid flows, the cell is deformed but its density obeys $D \sigma+\sigma \theta=0$, which in hyperbolic coordinates is equivalent to $\partial_{\mu}\left(\tau \sigma u^{\mu}\right)=0$. In terms of this reference density, the equations of motion used in this paper can be written as [27]

$$
\begin{aligned}
\gamma \frac{d}{d \tau}\left[\frac{(\varepsilon+p+\Pi)}{\sigma} u^{\mu}\right] & =\frac{1}{\sigma} \partial^{\mu}(p+\Pi) \\
\gamma \frac{d}{d \tau}\left(\frac{s}{\sigma}\right)+\left(\frac{\Pi}{\sigma}\right) \frac{\theta}{T} & =0 \\
\tau_{\Pi} \gamma \frac{d}{d \tau}\left(\frac{\Pi}{\sigma}\right)+\frac{\Pi}{\sigma}+\left(\frac{\zeta}{\sigma}\right) \theta & =0 .
\end{aligned}
$$

These equations are completely equivalent to those in Section $\llbracket$ but they are more suitable for the Lagrangian implementation via SPH, as will be explained in the following.

The fundamental idea behind a mesh free method such as SPH is that the boost invariant hydrodynamical fields can be reconstructed, at any given point in space and time, using a discrete set of Lagrangian coordinates $\left\{\mathbf{r}_{\alpha}(\tau), \alpha=1, \ldots, N_{S P H}\right\}$ together with a normalized piece-wise distribution function $W[\mathbf{r} ; h]$ for the discretization procedure. The kernel is chosen to have a finite support given by $h$, i.e, its value strictly vanishes for $|\mathbf{r}| \gg h$. Also, the kernel is a delta sequence in the sense that $\lim _{h \rightarrow 0} W[\mathbf{r} ; h]=\delta(\mathbf{r})$. The parameter $h$ is a length scale that represents the width of the kernel and it defines a cutoff for modes with shorter wavelength. The smaller the $h$ the larger is the number of SPH particles needed to accurately describe the flow. In general, the choice of $h$ dictates how much of the initial structure in the initial conditions will be reproduced and used as initial values for the subsequent dynamics. In practice, the actual size of $h$ is also limited by the computational time available. We shall discuss our choice for $h$ in more detail in the next section. For boost invariant hydrodynamics, the kernel function (in hyperbolic coordinates) is normalized as

$$
\int W[\mathbf{r} ; h] d^{2} \mathbf{r}=1
$$

The Kernel function used in v-USPhydro can be seen in Fig. 8

As was mentioned above, the conserved reference current density obeys the equation $\partial_{\mu}\left(\tau \sigma u^{\mu}\right)=0$ in hyperbolic coordinates. Within SPH, the reference density in the lab frame is expressed in Lagrangian coordinates as

$$
\tau \gamma \sigma \rightarrow \sigma^{*}(\mathbf{r}, \tau)=\sum_{\alpha=1}^{N_{S P H}} \nu_{\alpha} W\left[\mathbf{r}-\mathbf{r}_{\alpha}(\tau) ; h\right]
$$

where $\nu_{\alpha}$ are constants. Due to the normalization of the kernel, one can see that integral of the reference density in the transverse plane is a constant, i.e., $\int d^{2} \mathbf{r} \sigma^{*}(\mathbf{r}, \tau)=\sum_{\alpha=1}^{N_{S P H}} \nu_{\alpha}$. Therefore, it is natural to interpret the quantity $\nu_{\alpha}$ as a conserved quantity attached to the Lagrangian coordinate $\mathbf{r}_{\alpha}(\tau)$ and $\sigma^{*}$ as a sum of small piece-wise distributions $\nu_{\alpha} W\left[\mathbf{r}-\mathbf{r}_{\alpha}(\tau) ; h\right]$, which are called "SPH particles". 
One now defines the vector current

$$
\mathbf{j}^{*}(\mathbf{r}, \tau)=\sum_{\alpha=1}^{N_{S P H}} \nu_{\alpha} \frac{d \mathbf{r}_{\alpha}(\tau)}{d \tau} W\left[\mathbf{r}-\mathbf{r}_{\alpha}(\tau) ; h\right],
$$

so that the continuity equation $\partial_{\tau} \sigma^{*}(\mathbf{r}, \tau)+\nabla_{\mathbf{r}} \cdot \mathbf{j}^{*}(\mathbf{r}, \tau)=0$ for the reference density is automatically satisfied 33 . Eq. (A6) shows that each Lagrangian coordinate (or SPH particle) $\mathbf{r}_{\alpha}(\tau)$ has velocity $\mathbf{u}_{\alpha}(\tau)=\gamma_{\alpha}(\tau) \mathbf{v}_{\alpha}(\tau)$, where $\mathbf{v}_{\alpha}(\tau)=d \mathbf{r}_{\alpha}(\tau) / d \tau$ and $\gamma_{\alpha}=1 / \sqrt{1-\mathbf{v}_{\alpha}^{2}}$, and it carries a quantity $\nu_{\alpha}$ for the reference density $\sigma^{*}$.

Now, let $a(\mathbf{r}, \tau)$ be the density associated with some extensive quantity. The SPH description of this quantity is

$$
a(\mathbf{r}, \tau)=\sum_{\alpha=1}^{N_{S P H}} \nu_{\alpha} \frac{a\left(\mathbf{r}_{\alpha}(\tau)\right)}{\sigma^{*}\left(\mathbf{r}_{\alpha}(\tau)\right)} W\left[\mathbf{r}-\mathbf{r}_{\alpha}(\tau) ; h\right] .
$$

One can then see that any spatial gradient of $a(\mathbf{r}, \tau)$ acts only on the kernel function and this gradient is still a smooth function. For instance, for the zeroth component of the entropy current in the lab frame $s^{*}=s \gamma \tau$ one finds

$$
s^{*}(\mathbf{r}, \tau)=\sum_{\alpha=1}^{N_{S P H}} \nu_{\alpha} \frac{s\left(\mathbf{r}_{\alpha}(\tau)\right)}{\sigma\left(\mathbf{r}_{\alpha}(\tau)\right)} W\left[\mathbf{r}-\mathbf{r}_{\alpha}(\tau) ; h\right]
$$

while for the bulk term

$$
\Pi(\mathbf{r}, \tau)=\sum_{\alpha=1}^{N_{S P H}} \nu_{\alpha} \frac{1}{\gamma_{\alpha} \tau}\left(\frac{\Pi}{\sigma}\right)_{\alpha} W\left[\mathbf{r}-\mathbf{r}_{\alpha}(\tau) ; h\right] .
$$

The dynamical variables in the SPH method are then

$$
\left\{\mathbf{r}_{\alpha}, \mathbf{u}_{\alpha},\left(\frac{s}{\sigma}\right)_{\alpha},\left(\frac{\Pi}{\sigma}\right)_{\alpha} ; \alpha=1, . ., N_{S P H}\right\}
$$

and they represent the position, velocity, entropy, and bulk viscosity associated with the $\alpha$-th SPH particle, respectively. Using that $\partial_{\mu}\left(\tau \sigma u^{\mu}\right)=0$, we see that $\sigma^{*} \nabla \cdot \mathbf{v}=-\frac{d \sigma^{*}}{d \tau}$ and, consequently, the fluid expansion rate for each $\mathrm{SPH}$ particle is

$$
\theta_{\alpha}=\left(D_{\mu} u^{\mu}\right)_{\alpha}=\frac{d \gamma_{\alpha}}{d \tau}+\frac{\gamma_{\alpha}}{\tau}-\frac{\gamma_{\alpha}}{\sigma_{\alpha}^{*}} \frac{d \sigma_{\alpha}^{*}}{d \tau} .
$$

From the equations of motion in A1 w3 obtain the following equations associated with each SPH particle

$$
\begin{gathered}
\sigma^{*} \frac{d}{d \tau}\left(\frac{(\varepsilon+p+\Pi)_{\alpha}}{\sigma_{\alpha}} u_{i \alpha}\right)=\tau \sum_{\beta=1}^{N_{S P H}} \nu_{\beta} \sigma_{\alpha}^{*}\left(\frac{p_{\beta}+\Pi_{\beta}}{\left(\sigma_{\beta}^{*}\right)^{2}}+\frac{p_{\alpha}+\Pi_{\alpha}}{\left(\sigma_{\alpha}^{*}\right)^{2}}\right) \partial_{i} W\left[\mathbf{r}_{\alpha}-\mathbf{r}_{\beta}(\tau) ; h\right] \\
\gamma_{\alpha} \frac{d}{d \tau}\left(\frac{s}{\sigma}\right)_{\alpha}+\left(\frac{\Pi}{\sigma}\right)_{\alpha}\left(\frac{\theta}{T}\right)_{\alpha}=0
\end{gathered}
$$

and

$$
\tau_{\Pi_{\alpha}} \gamma_{\alpha} \frac{d}{d \tau}\left(\frac{\Pi}{\sigma}\right)_{\alpha}+\left(\frac{\Pi}{\sigma}\right)_{\alpha}+\left(\frac{\zeta}{\sigma}\right)_{\alpha} \theta_{\alpha}=0 .
$$

The r.h.s. of Eq. (A12) is the SPH representation of the gradients of pressure and bulk viscosity and, in the case of vanishing bulk viscosity, these equations become those found using the variational principle 33]. Eqs. (A12,A14) are the SPH representation of the equations of motion which are solved in $\mathrm{v}$-USPhydro. The beauty of the SPH method to solving hydrodynamics is that the coupled, nonlinear partial differential equations in the Eulerian view are described in terms of a set of nonlinear ordinary coupled differential equations for the Lagrangian variables.

We remark that in our Lagrangian approach no numerical viscosity is needed even in the inviscid case. Moreover, in our approach no extra conditions on the dynamical fields at very low temperatures needs to be imposed. In fact, we show below that our code in the absence of bulk viscosity exactly matches the analytical solution for $2+1$ inviscid conformal hydrodynamics derived by in 68], which provides a very stringent test of our approach to solve the equations of relativistic fluid dynamics. We have also checked that our code matches the calculations performed in [27]. 


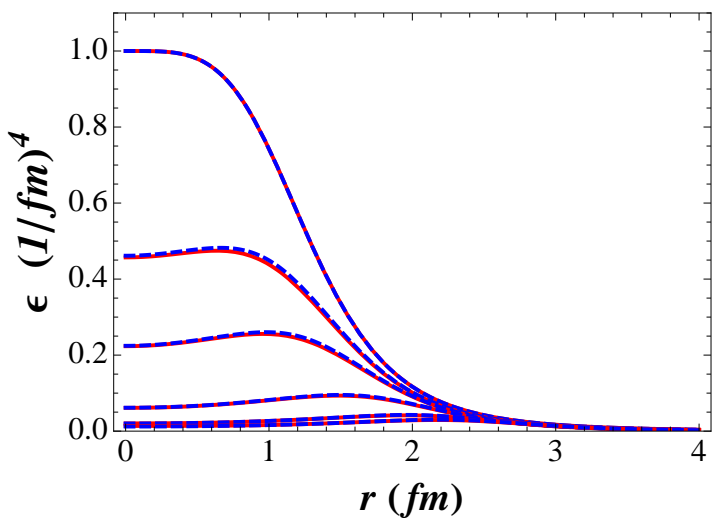

FIG. 9. Comparison between our numerical results for the energy density computed using v-USPhydro (dashed blue lines) and the analytical solution in Eq. A15), shown as solid red lines, for times $\tau=1,1.2,1.4,1.8,2.2$, and 2.4 fm. The energy density at the origin starts at $1 \mathrm{fm}^{-4}$ at $\tau_{0}=1 \mathrm{fm}$ and is reduced by an order of magnitude after $1.4 \mathrm{fm}$.

\section{Comparison to Gubser flow}

In [68], an analytical solution of $2+1$ (i.e., boost invariant) ideal conformal (i.e., $\varepsilon=3 p$ ) hydrodynamics was derived that can be used as a nontrivial check for the numerical hydrodynamic codes. They found the following analytical solution for the energy density profile

$$
\varepsilon(\tau, r)=\frac{\varepsilon_{0}}{\tau^{4 / 3}} \frac{(2 q)^{8 / 3}}{\left[1+2 q^{2}\left(\tau^{2}+r^{2}\right)+q^{4}\left(\tau^{2}-r^{2}\right)^{2}\right]^{4 / 3}}
$$

where

$$
r^{2}=x^{2}+y^{2}
$$

and $q$ (in $1 / \mathrm{fm}$ ) and $\varepsilon_{0}$ are constants set to 1 (the overall magnitude of the energy density, set by $\varepsilon_{0}$, is immaterial for this type of check). The analytical solution for the flow is [68]

$$
\begin{aligned}
& u_{x}(\tau, r)=\frac{\sinh [\kappa(\tau, r)] x}{r} \\
& u_{y}(\tau, r)=\frac{\sinh [\kappa(\tau, r)] y}{r}
\end{aligned}
$$

where

$$
\kappa(\tau, r)=\operatorname{arctanh}\left(\frac{2 q^{2} \tau r}{1+q^{2} \tau^{2}+q^{2} r^{2}}\right) .
$$

We take the analytical formulas shown above computed at $\tau_{0}=1 \mathrm{fm}$ to define the initial conditions for the energy density and flow. These initial conditions are used as input for our numerical code and we then compare our numerical results to the analytical solution for several values of $\tau$, as shown in Figs. 9, 10 ,

This system expands very rapidly, the energy density falls very steeply, and it requires a relatively small $h$, which in turn requires a great deal of SPH particles to achieve an accurate description of the analytical solution. v-USPhydro was able to match the analytical solution very well using $h=0.12 \mathrm{fm}$ and a total of $103041 \mathrm{SPH}$ particles, as seen in Figs. 9, 10, Note, however, that the Glauber initial conditions used in the numerical analysis in this paper do not expand as fast as this analytical solution (they also do not have initial transverse flow) and not as many SPH particles are needed in this case. The dependence of our results for the flow coefficients shown in Section $\nabla$ with the choice of $h$ and the total number of SPH particles is studied in Appendix [E.

\section{Appendix B: Some Details about the Cooper-Frye Freeze-Out within the SPH Approach}

In this section we present some details about the Cooper-Frye formalism employed in hadronic freeze-out 61. within the SPH approach [36, 39, 69]. In the Cooper-Frye freeze-out procedure [61], the particle flux through an 


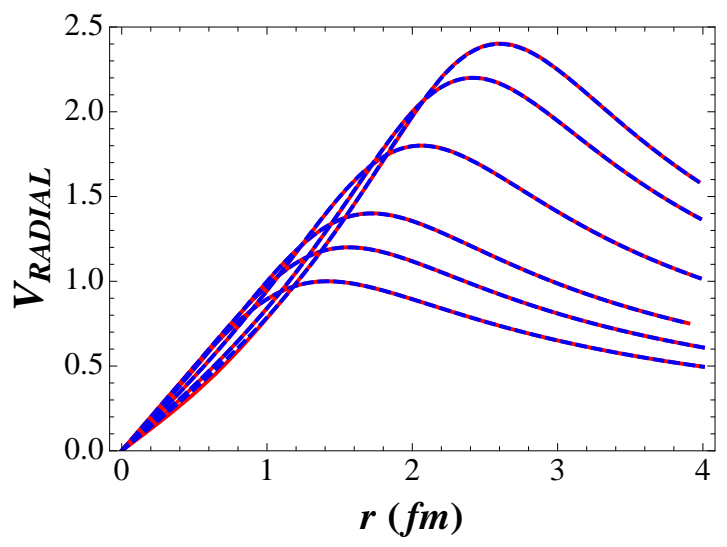

FIG. 10. Comparison between our numerical results for the radial velocity $\sqrt{u_{x}^{2}+u_{y}^{2}}$ computed using v-USPhydro (dashed blue lines) and the analytical solution in Eq. (A17), shown as solid red lines, for times $\tau=1,1.2,1.4,1.8,2.2$, and 2.4 fm. The initial transverse flow has a peak around 1 at $r \sim 1.4 \mathrm{fm}$ and peaks around 2.5 at $r \sim 2.6 \mathrm{fm}$ when $\tau=2.4 \mathrm{fm}$.

isothermal hypersurface $\Sigma$ defines the momentum distribution of a given particle species of degeneracy $g$, mass $m$, energy $E=\sqrt{p_{T}^{2}+m^{2}}$, and distribution function $f$

$$
\frac{d N}{d y p_{T} d p_{T} d \phi}=\frac{g}{(2 \pi)^{3}} \int_{\Sigma} d \Sigma \cdot p f\left(p \cdot u, \Pi, T_{F O}\right)
$$

where $T_{F O}$ is the freeze-out temperature, $y$ is the particle's rapidity, and $\Pi$ is the bulk viscosity contribution. Note that $p \cdot u$ and $\Pi$ are the only Lorentz invariant structures that need to be taken into account to describe the viscous effects coming solely from bulk viscosity.

As explained in [36, 39, 69], in the SPH formalism the integral over the isothermal hypersurface is written in terms of a sum of SPH particles as

$$
\frac{d N}{d y p_{T} d p_{T} d \phi}=\frac{g}{(2 \pi)^{3}} \sum_{\alpha=1}^{N_{S P H}} \frac{(p \cdot n)_{\alpha}}{(n \cdot u)_{\alpha}} \frac{\nu_{\alpha}}{\sigma_{\alpha}} f\left(T_{F O},(p \cdot u)_{\alpha}, \Pi_{\alpha}\right)
$$

where the index $\alpha$ indicates the SPH particle, $N_{S P H}$ is the total number of SPH particles, $\left(n_{\mu}\right)_{\alpha}$ is the normal vector of the isothermal hypersurface reconstructed using the $\alpha$-th SPH particle, $\left(u_{\mu}\right)_{\alpha}$ is the 4 -velocity of the SPH particle, and $\Pi_{\alpha}$ is the bulk viscosity of the SPH particle. The distribution function in the equation above is the sum of the ideal distribution function and the non-equilibrium correction $\delta f$ shown in Eq. (18) (for the sake of generality, here we do not use classical statistics).

In explicit form, the particle distribution for a given particle species is

$$
\frac{d N}{d y p_{T} d p_{T} d \phi}=\frac{g}{(2 \pi)^{3}} \sum_{\alpha=1}^{N_{S P H}}\left[q_{0 \alpha} \mathcal{I}_{1}\left(\alpha, m, T_{F O}\right)-\left(\mathbf{p}_{T} \cdot \mathbf{q}_{T}\right)_{\alpha} \mathcal{I}_{2}\left(\alpha, m, T_{F O}\right)\right]
$$

where

$$
\left(q_{\nu}\right)_{\alpha}=\frac{\left(n_{\nu}\right)_{\alpha}}{\left|(n \cdot u)_{\alpha}\right|} \frac{\nu_{\alpha}}{\sigma_{\alpha}}
$$

and

$$
\begin{aligned}
\mathcal{I}_{1}\left(\alpha, m, T_{F O}\right) & =2 \sum_{n=0}^{\infty}(-a)^{n} \lambda_{\alpha}^{n+1}\left\{E^{2} F_{\alpha}^{(1)}(n) K_{0}\left[\frac{(n+1) E \gamma_{\alpha}}{T_{F O}}\right]\right. \\
& \left.+K_{1}\left[\frac{(n+1) E \gamma_{\alpha}}{T_{F O}}\right] E\left(F_{\alpha}^{(0)}(n)+\frac{F_{\alpha}^{(1)}(n) T_{F O}}{(n+1) \gamma_{\alpha}}+E^{2} F_{\alpha}^{(2)}(n)\right)+\frac{E^{2} F_{\alpha}^{(2)}(n) T_{F O}}{(n+1) \gamma_{\alpha}} K_{2}\left[\frac{(n+1) E \gamma_{\alpha}}{T_{F O}}\right]\right\}
\end{aligned}
$$


and

$$
\begin{aligned}
\mathcal{I}_{2}\left(\alpha, m, T_{F O}\right) & =2 \sum_{n=0}^{\infty}(-a)^{n} \lambda_{\alpha}^{n+1}\left\{K_{0}\left[\frac{(n+1) E \gamma_{\alpha}}{T_{F O}}\right]\left(F_{\alpha}^{(0)}(n)+F_{\alpha}^{(2)}(n) E^{2}\right)\right. \\
& \left.+E K_{1}\left[\frac{(n+1) E \gamma_{\alpha}}{T_{F O}}\right]\left(F_{\alpha}^{(1)}(n)+\frac{F_{\alpha}^{(2)}(n) T_{F O}}{(n+1) \gamma_{\alpha}}\right)\right\}
\end{aligned}
$$

with $a=1(-1)$ for fermions(bosons) and 0 for classical particles, $\lambda_{\alpha}=e^{\left(\mathbf{p}_{T} \cdot \mathbf{u}_{T}\right)_{\alpha} / T_{F O}}, K_{n}[x]$ is a modified Bessel function, and

$$
\begin{gathered}
F_{\alpha}^{(0)}(n)=1+(n+1) \Pi_{\alpha}^{p i o n}\left[B_{0}-D_{0}\left(\mathbf{p}_{T} \cdot \mathbf{u}_{T}\right)_{\alpha}+E_{0}\left(\mathbf{p}_{T} \cdot \mathbf{u}_{T}\right)_{\alpha}^{2}\right] \\
F_{\alpha}^{(1)}(n)=(n+1) \Pi_{\alpha} \gamma_{\alpha}\left[D_{0}-2 E_{0}\left(\mathbf{p}_{T} \cdot \mathbf{u}_{T}\right)_{\alpha}\right] \\
F_{\alpha}^{(2)}(n)=(n+1) \Pi_{\alpha} \gamma_{\alpha}^{2} E_{0}
\end{gathered}
$$

\section{Appendix C: Event Plane Method for Collective Flow Coefficients}

We use the event plane method to compute the collective flow coefficients [66]. First, we use that given the differential number $\frac{d N_{i}\left(p_{T}, \phi\right)}{d y p_{T} d p_{T} d \phi}$ of hadrons of species $i$ in a given event, we can integrate it over the azimuthal angle $\phi$ to find the spectrum

$$
\frac{d N_{i}\left(p_{T}\right)}{d y p_{T} d p_{T}}=\int_{0}^{2 \pi} d \phi \frac{d N_{i}}{d y p_{T} d p_{T} d \phi}
$$

We then define the event plane vectors

$$
\begin{gathered}
Q_{x}^{i}[n]=\int_{p_{T \text { min }}}^{p_{T \text { max }}} d p_{T} p_{T}^{2} \int_{0}^{2 \pi} d \phi \cos (n \phi) \frac{d N_{i}}{d y p_{T} d p_{T} d \phi} \\
Q_{y}^{i}[n]=\int_{p_{\text {T min }}}^{p_{T \text { max }}} d p_{T} p_{T}^{2} \int_{0}^{2 \pi} d \phi \sin (n \phi) \frac{d N_{i}}{d y p_{T} d p_{T} d \phi}
\end{gathered}
$$

and the event plane angles

$$
\psi^{i}[n]=\frac{1}{n} \tan ^{-1}\left(\frac{Q_{y}^{i}[n]}{Q_{x}^{i}[n]}\right),
$$

where in this paper $p_{T \min }=0 \mathrm{GeV}$ and $p_{T \max }=3 \mathrm{GeV}$.

The collective flow coefficients as functions of the transverse momentum are then

$$
v_{n}^{i}\left(p_{T}\right)=\frac{\int_{0}^{2 \pi} d \phi \frac{d N_{i}}{d y p_{T} d p_{T} d \phi} \cos \left[n\left(\phi-\psi^{i}[n]\right)\right]}{\frac{d N_{i}\left(p_{T}\right)}{d y p_{T} d p_{T}}} .
$$

The integrated $v_{n}$ 's are given by

$$
v_{n}^{i}=\frac{\int_{p_{T \min }}^{p_{T \max }} d p_{T} p_{T} \int_{0}^{2 \pi} d \phi \frac{d N_{i}}{d y p_{T} d p_{T} d \phi} \cos \left[n\left(\phi-\psi^{i}[n]\right)\right]}{\int_{p_{T \min }}^{p_{T} \max } d p_{T} p_{T} \frac{d N_{i}\left(p_{T}\right)}{d y p_{T} d p_{T}}} .
$$




\section{Appendix D: Formulas for the Coefficients in $\delta f$}

In this paper, $\delta f_{\mathbf{k}}^{(i)}$ is computed following the steps outlined in Refs. [50, 63]. In this Appendix, we just outline how the coefficients that appear in $\delta f_{\mathbf{k}}^{(i)}$ can be computed.

The coefficient $a_{n r}^{(0) i}$ is a function of the temperature and the mass of the $i$-th hadron species,

$$
\begin{aligned}
\frac{a_{10}^{(0) i}}{a_{11}^{(0) i}} & =-\frac{J_{10}^{i}}{J_{00}^{i}} \\
\left(a_{11}^{(0) i}\right)^{2} & =\frac{\left(J_{00}^{i}\right)^{2}}{J_{20}^{i} J_{00}^{i}-\left(J_{10}^{i}\right)^{2}}, \\
\frac{a_{21}^{(0) i}}{a_{22}^{(0) i}} & =\frac{J_{20}^{i} J_{10}^{i}-J_{30}^{i} J_{00}^{i}}{J_{20}^{i} J_{00}^{i}-\left(J_{10}^{i}\right)^{2}} \\
\frac{a_{20}^{(0) i}}{a_{22}^{(0) i}} & =\frac{J_{10}^{i} J_{30}^{i}-\left(J_{20}^{i}\right)^{2}}{J_{00}^{i} J_{20}^{i}-\left(J_{10}^{i}\right)^{2}}, \\
\left(a_{22}^{(0) i}\right)^{2} & =J_{00}^{i}\left(J_{40}^{i}-\frac{\left(J_{20}^{i}\right)^{3}-2 J_{30}^{i} J_{20}^{i} J_{10}^{i}+\left(J_{30}^{i}\right)^{2} J_{00}^{i}}{J_{20}^{i} J_{00}^{i}-\left(J_{10}^{i}\right)^{2}} .\right.
\end{aligned}
$$

The thermodynamic functions $J_{n q}^{i}$ are defined as

$$
J_{n q}^{i}=g_{i} \int \frac{d^{3} \mathbf{k}}{(2 \pi)^{3} k_{i}^{0}}\left(u \cdot k_{i}\right)^{n-2 q}\left(-\Delta_{\alpha \beta} k_{i}^{\alpha} k_{i}^{\beta}\right)^{q} f_{0 \mathbf{k}}^{i}\left(1-a f_{0 \mathbf{k}}^{i}\right)
$$

where $g_{i}$ is the degeneracy factor, $d K_{i} \equiv d^{3} \mathbf{k} /\left[(2 \pi)^{3} k_{i}^{0}\right]$, and $f_{0 \mathbf{k}}^{i}$ is the local equilibrium distribution function

$$
f_{0 \mathbf{k}}^{i}=\frac{1}{\exp \left(\beta u \cdot k_{i}\right)+a}
$$

with $a$ being $a=1(-1)$ for fermions(bosons), and 0 for classical particles, and we assumed that the chemical potential is zero. For completeness, we shall also define the thermodynamic function $I_{n q}^{i}$

$$
I_{n q}^{i}=g_{i} \int \frac{d^{3} \mathbf{k}}{(2 \pi)^{3} k_{i}^{0}}\left(E_{i \mathbf{k}}\right)^{n-2 q}\left(-\Delta_{\alpha \beta} k_{i}^{\alpha} k_{i}^{\beta}\right)^{q} f_{0 \mathbf{k}}^{i}
$$

where we defined $E_{i \mathbf{k}} \equiv u \cdot k_{i}$. In this paper we only consider classical particles for constructing an approximate expression for the $\delta f_{\mathbf{k}}^{i}$ of hadrons. Therefore, the functions $I_{n q}^{i}$ and $J_{n q}^{i}$ are equivalent.

The coefficients $\alpha_{i, m}$ are given by

$$
\left(\begin{array}{c}
\alpha_{i, 0} \\
\alpha_{i, 2}
\end{array}\right)=\hat{\mathcal{M}}_{(0)}^{-1}\left(\begin{array}{c}
\beta_{i, 0} \\
\beta_{i, 2}
\end{array}\right)
$$

where

$$
\begin{aligned}
& \beta_{i, r}=J_{r 0}^{i} \frac{J_{30} n_{0}-J_{20}\left(\varepsilon_{0}+P_{0}\right)}{J_{30} J_{10}-J_{20} J_{20}} \theta-J_{r+1,0}^{i} \frac{J_{20} n_{0}-J_{10}\left(\varepsilon_{0}+P_{0}\right)}{J_{30} J_{10}-J_{20} J_{20}} \theta-\left[(r-1) I_{r 1}^{i}+I_{r 0}^{i}\right] \theta, \\
& J_{n q}=\sum_{i=1}^{N} J_{n q}^{i}, I_{n q}=\sum_{i=1}^{N} I_{n q}^{i} .
\end{aligned}
$$

Above, $\hat{\mathcal{M}}_{(0)}$ is a $(2 N-1) \times(2 N-1)$ matrix that can be derived from the collision term of the Boltzmann equation. 
Since we consider only elastic 2-to-2 collisions, it can be simplified to be

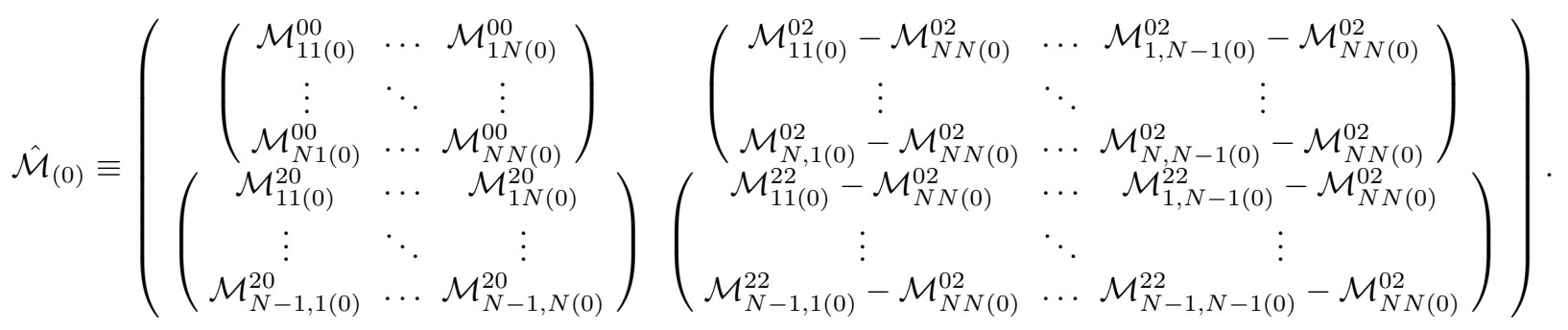

where

$$
\mathcal{M}_{i j}^{r n}=\mathcal{A}_{r n}^{(i 0)} \delta_{i j}+\mathcal{C}_{r n}^{(i j 0)}
$$

and

$$
\begin{aligned}
\mathcal{A}_{r n}^{(i 0)} & =\frac{g_{i} g_{j}}{J_{00}^{i}} \sum_{j=1}^{N} \int d K_{i} d K_{j}^{\prime} d P_{i} d P_{j}^{\prime} \gamma_{i j} W_{\mathbf{p p}^{\prime}-\mathbf{k} \mathbf{k}^{\prime}}^{i j} f_{i \mathbf{k}}^{(0)} f_{j \mathbf{k}^{\prime}}^{(0)}\left(E_{i \mathbf{k}}\right)^{r}\left[h_{n i}^{(1)}\left(E_{\mathbf{p} i}-E_{\mathbf{k} i}\right)+h_{n i}^{(2)}\left(E_{\mathbf{p}_{i}}^{2}-E_{\mathbf{k} i}^{2}\right)\right] \\
\mathcal{C}_{r n}^{(i j 0)} & =\frac{g_{i} g_{j}}{J_{00}^{i}} \int d K_{i} d K_{j}^{\prime} d P_{i} d P_{j}^{\prime} \gamma_{i j} W_{\mathbf{p p}^{\prime}-\mathbf{k} \mathbf{k}^{\prime}}^{i j} f_{i \mathbf{k}}^{(0)} f_{j \mathbf{k}^{\prime}}^{(0)}\left(E_{i \mathbf{k}}\right)^{r} \times\left[h_{n j}^{(1)}\left(E_{\mathbf{p}^{\prime} j}-E_{\mathbf{k}^{\prime} j}\right)+h_{n j}^{(2)}\left(E_{\mathbf{p}^{\prime} j}^{2}-E_{\mathbf{k}^{\prime} j}^{2}\right)\right]
\end{aligned}
$$

with $W_{\mathbf{p p}^{\prime}-\mathbf{k k}^{\prime}}^{i j}=s \sigma_{i j}(2 \pi)^{6} \delta^{4}\left(p_{i}+p_{j}^{\prime}-k_{i}-k_{j}^{\prime}\right)$ being the transition rate, $N$ is the number of hadrons considered, $\gamma_{i j}=1-(1 / 2) \delta_{i j}, s$ is the Mandelstan variable, and we defined,

$$
\begin{aligned}
& h_{0 i}^{(0)}=1+a_{10}^{(0) i} a_{10}^{(0) i}+a_{20}^{(0) i} a_{20}^{(0) i}, \\
& h_{1 i}^{(0)}=a_{11}^{(0) i} a_{10}^{(0) i}+a_{20}^{(0) i} a_{21}^{(0) i}, \\
& h_{2 i}^{(0)}=a_{20}^{(0) i} a_{22}^{(0) i}, \\
& h_{0 i}^{(1)}=a_{10}^{(0) i} a_{11}^{(0) i}+a_{20}^{(0) i} a_{21}^{(0) i}, \\
& h_{1 i}^{(1)}=a_{11}^{(0) i} a_{11}^{(0) i}+a_{21}^{(0) i} a_{21}^{(0) i}, \\
& h_{2 i}^{(1)}=a_{21}^{(0) i} a_{22}^{(0) i}, \\
& h_{0 i}^{(2)}=a_{20}^{(0) i} a_{22}^{(0) i}, \\
& h_{1 i}^{(2)}=a_{21}^{(0) i} a_{22}^{(0) i}, \\
& h_{2 i}^{(2)}=a_{22}^{(0) i} a_{22}^{(0) i} .
\end{aligned}
$$

\section{Appendix E: Convergence of the Results with the Choice of $h$ and the Number of SPH Particles}

In this paper, the effects of bulk viscosity on collective flow coefficients were computed using v-USPhydro with $h=0.3 \mathrm{fm}$ and a total number of SPH particles $\left(N_{S P H}\right)$ of roughly $3 \times 10^{4}$. However, it is important to test the convergence of our results with the choice of these parameters, i.e, if one were to include more SPH particles would there be a change in the flow harmonics and if so by what percentage? To demonstrate this we first set $h=0.3 \mathrm{fm}$ and vary the number of SPH particles using averaged initial conditions in the $20-30 \%$ centrality bin. The initial condition was averaged over roughly 150 events, which means that it is mostly smooth but there is still some small remaining structure that generates small, though nonzero, higher order flow harmonics. In Fig. 11]we show our results for both the $v_{n}\left(p_{T}\right)$ 's and the relative percentage difference defined as

$$
v_{n} \%\left(N_{S P H}\right)=100\left|\frac{v_{n}\left(N_{S P H}\right)-v_{n}\left(N_{\infty}\right)}{v_{n}\left(N_{\infty}\right)}\right|
$$

where $N_{\infty}=159600 \mathrm{SPH}$ particles is the maximum number of particles set by our computational limitations.

As one can see in Fig. 11] our choice of about $27000 \mathrm{SPH}$ appears reasonable with the corresponding $h=0.3 \mathrm{fm}$ (at very low $p_{T}$ there is a greater deviation because the $v_{n}$ 's approach zero, which makes the comparison more difficult). Quite generally, the different choices for $N_{S P H}$ differ from the "infinite" $N_{\infty}$ limit by only $2-5 \%$ depending on the 

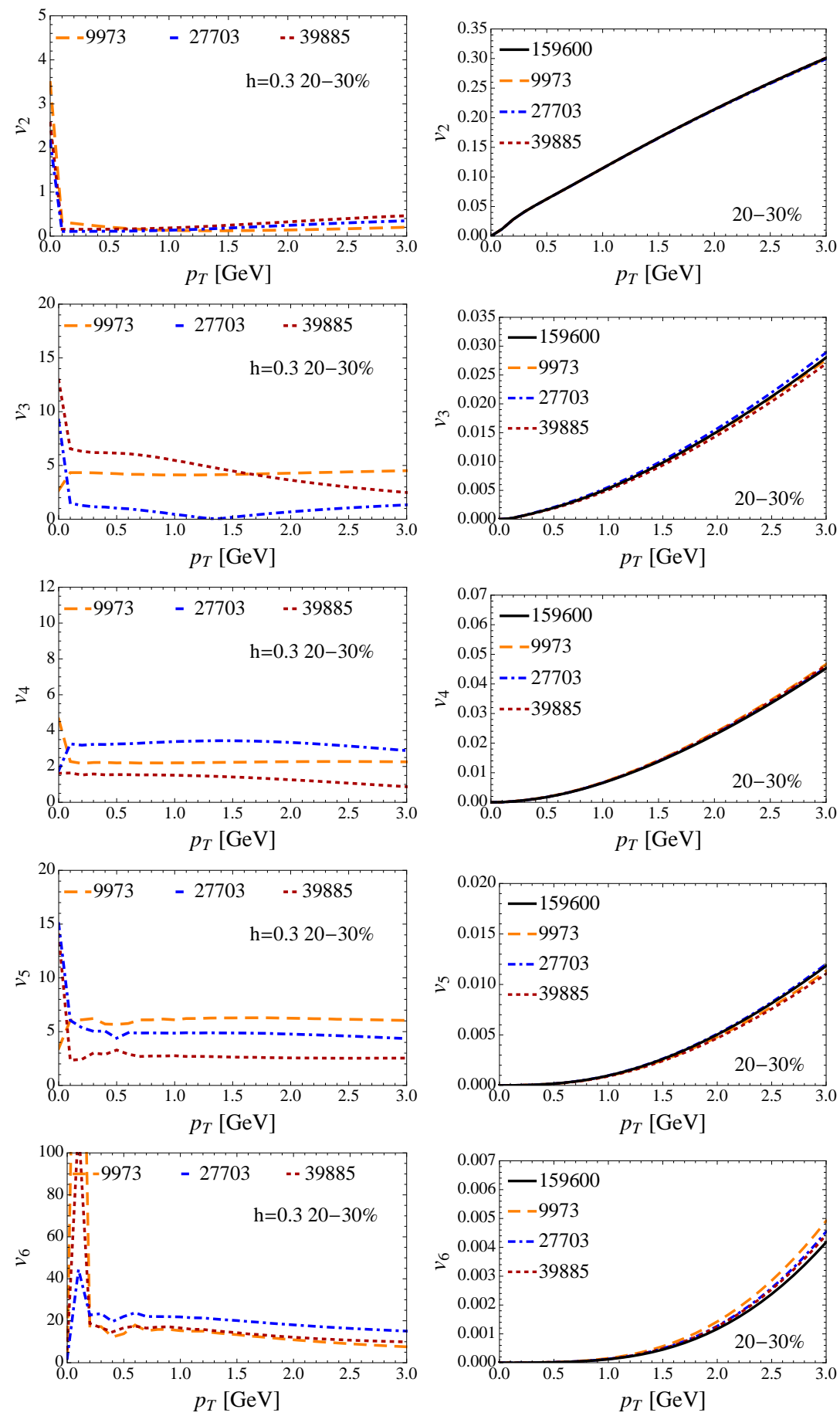

FIG. 11. Study of the convergence of the $v_{n}\left(p_{T}\right)$ coefficients with the number of SPH particles for a fixed $h=0.3 \mathrm{fm}$. On the left we show the percentage deviation, see Eq. (E1), with respect to our maximum of $159600 \mathrm{SPH}$ particles. On the right we show the actual value of the $v_{n}\left(p_{T}\right)$ 's for the different $N_{S P H}$ values. We used a single optical Glauber initial condition averaged over 150 events in the $20-30 \%$ centrality class. 
specific $v_{n}\left(p_{T}\right)$ and the difference is practically indiscernible with the naked eye (see the right plot in Fig. 11). Elliptic flow is found to be by far the most robust. The one exception being $v_{6}\left(p_{T}\right)$, which after this study we have decided not to include in the paper in the results section due to the large deviation. To perform a reliable study of $v_{6}\left(p_{T}\right)$ an extremely large number of SPH particles would be needed, which would significantly slow down computation time.

Additionally, we test the convergence of our results with our choice for $h$. Because $h$ is a smoothing parameter, if we choose $h=0.3 \mathrm{fm}$ this inherently limits our ability to probe very short length scales. Thus, it is important that $h$ is small enough to take into account necessary fluctuations but also large enough to allow for a reasonable computational time. We show the variation of our results for the flow coefficients with the choice of $h$ in Fig. 12 . The left plot shows the percentage difference with respect to the $N_{\infty}$ limit and $h=0.1$ fm while the right plot shows the actual values of the coefficients versus $p_{T}$ for various values of $h$ and $N_{S P H}$. One can see in Fig. 12 that the difference between $h=0.5 \mathrm{fm}$ and $h=0.3 \mathrm{fm}$ is not that large, however, results for $h=0.7$ fm consistently show a larger deviation for $v_{2}$ to $v_{5}$. This suggests that for the averaged Glauber initial conditions used in these tests most of the important structure is larger than $0.5 \mathrm{fm}$. Clearly, if one were to consider other types of initial conditions which display structure at smaller length scales, such as those that include gluon saturation effects [56], the type of analysis discussed in this Appendix must be performed again.

[1] M. Gyulassy, L. McLerran, Nucl. Phys. A 750, 30 (2005) nucl-th/0405013.

[2] For a recent review, see U. W. Heinz, R. Snellings, arXiv:1301.2826 [nucl-th].

[3] P. Danielewicz, M. Gyulassy, Phys. Rev. D 31, 53 (1985).

[4] P. Kovtun, D. T. Son and A. O. Starinets, Phys. Rev. Lett. 94, 111601 (2005) hep-th/0405231.

[5] P. B. Arnold, G. D. Moore, L. G. Yaffe, JHEP 0011, 001 (2000) hep-ph/0010177.

[6] P. B. Arnold, G. D. Moore, L. G. Yaffe, JHEP 0305, 051 (2003) hep-ph/0302165.

[7] M. Prakash, M. Prakash, R. Venugopalan and G. Welke, Phys. Rep. 227, 321 (1993).

[8] T. Hirano and M. Gyulassy, Nucl. Phys. A 769, 71 (2006) nucl-th/0506049.

[9] L. P. Csernai, J. .I. Kapusta, L. D. McLerran, Phys. Rev. Lett. 97, 152303 (2006) nucl-th/0604032].

[10] J. Noronha-Hostler, J. Noronha and C. Greiner, Phys. Rev. Lett. 103, 172302 (2009) arXiv:0811.1571 [nucl-th]].

[11] B. Schenke, S. Jeon, C. Gale, Phys. Rev. Lett. 106, 042301 (2011) arXiv:1009.3244 [hep-ph]].

[12] B. Schenke, S. Jeon, C. Gale, Phys. Rev. C 85, 024901 (2012) arXiv:1109.6289 [hep-ph]].

[13] P. B. Arnold, C. Dogan, G. D. Moore, Phys. Rev. D 74, 085021 (2006) hep-ph/0608012.

[14] A. Dobado, F. J. Llanes-Estrada and J. M. Torres-Rincon, Phys. Lett. B 702, 43 (2011) arXiv:1103.0735 [hep-ph]].

[15] E. Lu, G. D. Moore, Phys. Rev. C 83, 044901 (2011) arXiv:1102.0017 [hep-ph]].

[16] S. Borsanyi, G. Endrodi, Z. Fodor, A. Jakovac, S. D. Katz, S. Krieg, C. Ratti, K. K. Szabo, JHEP 1011, 077 (2010) arXiv:1007.2580 [hep-lat]].

[17] D. Kharzeev, K. Tuchin, JHEP 0809, 093 (2008) arXiv:0705.4280 [hep-ph]].

[18] F. Karsch, D. Kharzeev, K. Tuchin, Phys. Lett. B 663, 217 (2008) arXiv:0711.0914 [hep-ph]].

[19] V. Ozvenchuk, O. Linnyk, M. I. Gorenstein, E. L. Bratkovskaya and W. Cassing, arXiv:1212.5393 [hep-ph].

[20] S. S. Gubser, S. S. Pufu, F. D. Rocha, JHEP 0808, 085 (2008) arXiv:0806.0407 [hep-th]].

[21] G. Torrieri, B. Tomasik, I. Mishustin, Phys. Rev. C 77, 034903 (2008) arXiv:0707.4405 [nucl-th]].

[22] G. Torrieri, I. Mishustin, Phys. Rev. C 78, 021901 (2008) arXiv:0805.0442 [hep-ph]].

[23] G. Basar, D. Kharzeev, V. Skokov, Phys. Rev. Lett. 109, 202303 (2012) arXiv:1206.1334 [hep-ph]].

[24] A. Monnai, T. Hirano, Phys. Rev. C 80, 054906 (2009) arXiv:0903.4436 [nucl-th]].

[25] H. Song, U. W. Heinz, Phys. Rev. C 81, 024905 (2010) arXiv:0909.1549 [nucl-th]].

[26] P. Bozek, Phys. Rev. C 81, 034909 (2010) arXiv:0911.2397 [nucl-th]].

[27] G. S. Denicol, T. Kodama, T. Koide and P. Mota, Phys. Rev. C 80, 064901 (2009) arXiv:0903.3595 [hep-ph]]; G. S. Denicol, T. Kodama and T. Koide, J. Phys. G 37, 094040 (2010) [arXiv:1002.2394 [nucl-th]].

[28] V. Roy, A. K. Chaudhuri, Phys. Rev. C 85, 024909 (2012) [Erratum-ibid. C 85, 049902 (2012)] [arXiv:1109.1630 [nucl-th]].

[29] K. Dusling, T. Schäfer, Phys. Rev. C 85, 044909 (2012) arXiv:1109.5181 [hep-ph]].

[30] P. Bozek and W. Broniowski, Phys. Rev. C 85, 044910 (2012) arXiv:1203.1810 [nucl-th]].

[31] L. B. Lucy, ApJ. 82, 1013 (1977); R. A. Gingold and J. J. Monaghan, MNRAS 181, 375 (1977); R. A. Gingold and J. J. Monaghan, Mon. Not. Roy. Astron. Soc. 181, 375 (1977).

[32] J. J. Monaghan, Annu. Rev. Astron. Astrophys. 30, 543 (1992); E. Chow and J. J. Monaghan, J. Comput. Phys. 134, 296 (1997); P. Laguna, W. A. Miller and W. H. Zurek, Phys. Rev. D 41, 451 (1990); R. J. Thacker, E. R. Tittley, F. R. Pearce, H. M. P. Couchman and P. A. Thomas, Mon. Not. Roy. Astron. Soc. 319, 619 (2000) astro-ph/9809221.

[33] C. E. Aguiar, T. Kodama, T. Osada and Y. Hama, J. Phys. G 27, 75 (2001) hep-ph/0006239.

[34] V. Springel, Mon. Not. Roy. Astron. Soc. 364, 1105 (2005) astro-ph/0505010.

[35] G. R. Liu, M. B. Liu, "Smoothed Particle Hydrodynamics: A Meshfree Particle Method", 472 pages, World Scientific, 2003, ISBN: 981-238-456-1; D. Violeau, "Fluid Mechanics and the SPH Method - Theory and Applications", 616 pages, Oxford University Press, 2012, ISBN-10: 0199655529.

[36] T. Osada, C. E. Aguiar, Y. Hama and T. Kodama, nucl-th/0102011 

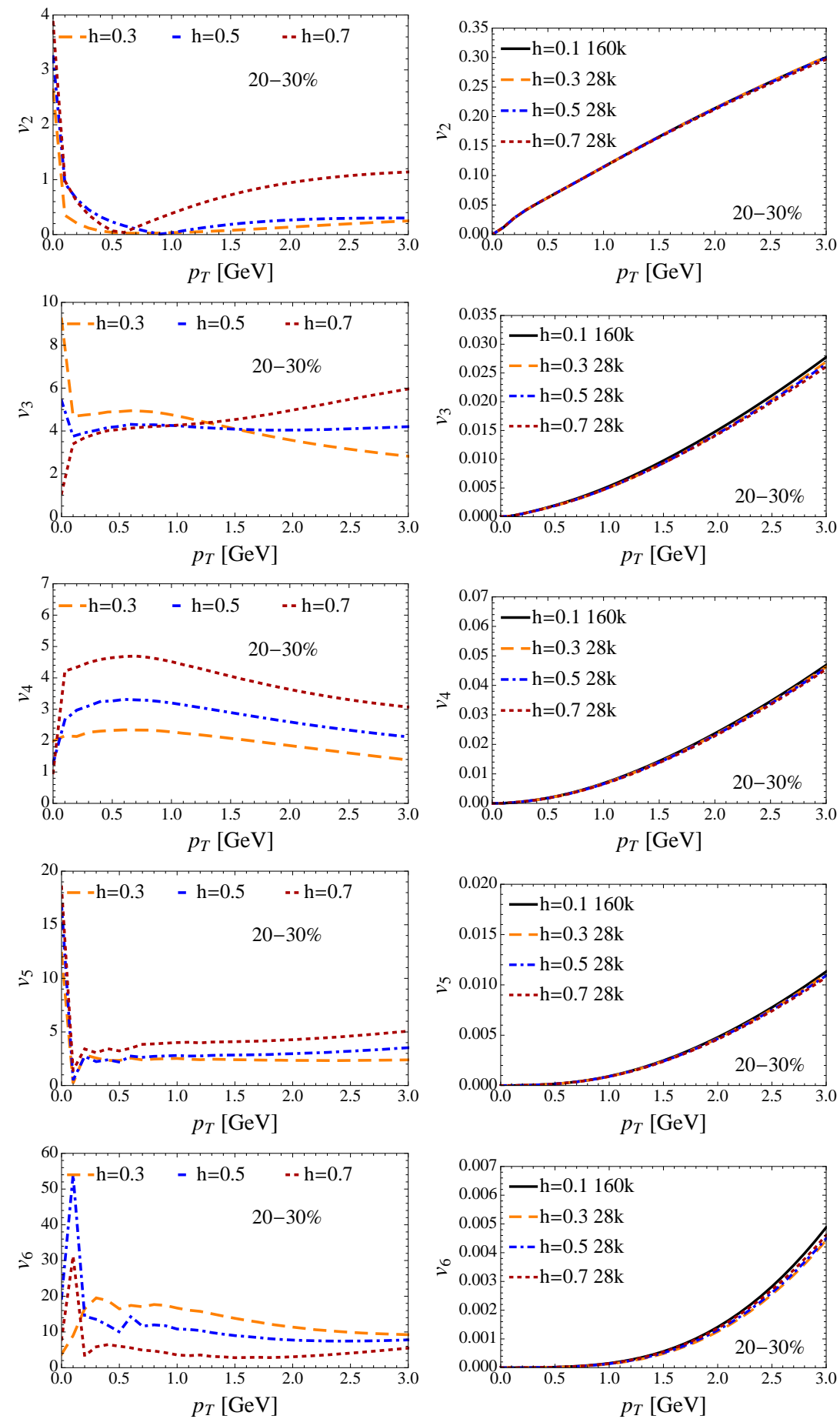

FIG. 12. The effect of the choice of $h$ on the total convergence of the $v_{n}$ 's $(h=0.3 \mathrm{fm}, h=0.5 \mathrm{fm}$, and $h=0.7 \mathrm{fm})$. On the left the percentage deviation (see Eq. (E1) is shown compared to $h=0.1 \mathrm{fm}$ for $159,600 \mathrm{SPH}$ particles. On the right we show the $v_{n}\left(p_{T}\right)$ 's varying $N_{S P H}$ and $h$. We used a single optical Glauber initial condition averaged over 150 events in the $20-30 \%$ centrality class. 
[37] C. E. Aguiar, Y. Hama, T. Kodama and T. Osada, Nucl. Phys. A 698, 639 (2002) hep-ph/0106266.

[38] O. Socolowski, Jr., F. Grassi, Y. Hama and T. Kodama, Phys. Rev. Lett. 93, 182301 (2004) hep-ph/0405181.

[39] Y. Hama, T. Kodama and O. Socolowski, Jr., Braz. J. Phys. 35, 24 (2005) hep-ph/0407264.

[40] Y. Hama, R. P. G. Andrade, F. Grassi, O. Socolowski, Jr., T. Kodama, B. Tavares and S. S. Padula, Nucl. Phys. A 774, 169 (2006) hep-ph/0510096.

[41] R. Andrade, F. Grassi, Y. Hama, T. Kodama and O. Socolowski, Jr., Phys. Rev. Lett. 97, 202302 (2006) nucl-th/0608067.

[42] R. P. G. Andrade, F. Grassi, Y. Hama, T. Kodama and W. L. Qian, Phys. Rev. Lett. 101, 112301 (2008) arXiv:0805.0018 [hep-ph]].

[43] J. Takahashi, B. M. Tavares, W. L. Qian, R. Andrade, F. Grassi, Y. Hama, T. Kodama and N. Xu, Phys. Rev. Lett. 103, 242301 (2009) arXiv:0902.4870 [nucl-th]].

[44] R. Derradi de Souza, J. Takahashi, T. Kodama and P. Sorensen, Phys. Rev. C 85, 054909 (2012) arXiv:1110.5698 [hep-ph]].

[45] F. G. Gardim, F. Grassi, M. Luzum and J. -Y. Ollitrault, Phys. Rev. C 85, 024908 (2012) arXiv:1111.6538 [nucl-th]].

[46] F. G. Gardim, F. Grassi, M. Luzum and J. -Y. Ollitrault, Phys. Rev. Lett. 109, 202302 (2012) [arXiv:1203.2882 [nucl-th]].

[47] F. G. Gardim, F. Grassi, M. Luzum and J. -Y. Ollitrault, Phys. Rev. C 87, 031901, (2013) (R) arXiv:1211.0989 [nucl-th]].

[48] G. S. Denicol, T. Kodama, T. Koide and P. Mota, J. Phys. G 35, 115102 (2008) arXiv:0807.3120 [hep-ph]].

[49] G. S. Denicol, T. Kodama, T. Koide and P. Mota, arXiv:0808.3170 [hep-ph].

[50] G. S. Denicol, H. Niemi, E. Molnar and D. H. Rischke, Phys. Rev. D 85, 114047 (2012) arXiv:1202.4551 [nucl-th]].

[51] C. Nonaka, S. A. Bass, Phys. Rev. C 75, 014902 (2007) nucl-th/0607018; C. Nonaka, E. Honda, S. Muroya, Eur. Phys. J. C 17, 663 (2000) hep-ph/0007187.

[52] H. -J. Drescher and Y. Nara, Phys. Rev. C 75, 034905 (2007) nucl-th/0611017; Phys. Rev. C 76, 041903 (2007) arXiv:0707.0249 [nucl-th]].

[53] S. S. Adler et al. [PHENIX Collaboration], Phys. Rev. C 69, 034909 (2004) nucl-ex/0307022.

[54] I. Arsene et al. [BRAHMS Collaboration], Nucl. Phys. A 757, 1 (2005) nucl-ex/0410020.

[55] J. Noronha-Hostler, "Properties of Hadronic Matter Near the Phase Transition", PhD thesis, Goethe University, Frankfurt , 2010.

[56] C. Gale, S. Jeon, B. Schenke, P. Tribedy, R. Venugopalan and, Phys. Rev. Lett. 110, 012302 (2013) arXiv:1209.6330 [nucl-th]].

[57] J. S. Moreland, Z. Qiu, U. W. Heinz, arXiv:1210.5508 [nucl-th].

[58] P. Huovinen and P. Petreczky, Nucl. Phys. A 837, 26 (2010) arXiv:0912.2541 [hep-ph]].

[59] A. Buchel, Phys. Lett. B 663, 286 (2008) arXiv:0708.3459 [hep-th]].

[60] X. -G. Huang, T. Kodama, T. Koide and D. H. Rischke, Phys. Rev. C 83, 024906 (2011) arXiv:1010.4359 [nucl-th]].

[61] F. Cooper and G. Frye, Phys. Rev. D 10, 186 (1974).

[62] J. Noronha-Hostler, J. Noronha, G. S. Denicol, R. P. G. Andrade, F. Grassi, C. Greiner, arXiv:1302.7038 [nucl-th].

[63] G. S. Denicol and H. Niemi, arXiv:1212.1473 [nucl-th].

[64] G. S. Denicol, H. Niemi, I. Bouras, E. Molnar, Z. Xu, D. H. Rischke and C. Greiner, arXiv:1207.6811 [nucl-th].

[65] M. Greif, F. Reining, I. Bouras, G. S. Denicol, Z. Xu and C. Greiner, Phys. Rev. E 87, 033019 (2013) arXiv:1301.1190 [hep-ph]].

[66] A. M. Poskanzer and S. A. Voloshin, Phys. Rev. C 58, 1671 (1998) nucl-ex/9805001].

[67] K. Dusling, G. D. Moore, D. Teaney, Phys. Rev. C 81, 034907 (2010) arXiv:0909.0754 [nucl-th]].

[68] S. S. Gubser, Phys. Rev. D 82, 085027 (2010) [arXiv:1006.0006 [hep-th]].

[69] G. S. Denicol, "Efeitos da Viscosidade no Escoamento da Matéria em Processos Nucleares Relativísticos", Master's Thesis, UFRJ, 2009. 